\title{
Multiwavelength study of the low-luminosity outbursting young star HBC $722^{\star}$
}

\author{
Á. Kóspál ${ }^{1}$, P. Ábrahám ${ }^{1}$, J. A. Acosta-Pulido ${ }^{2,3}$, M. M. Dunham ${ }^{4}$, D. García-Álvarez ${ }^{2,3,5}$, M. R. Hogerheijde ${ }^{6}$, \\ M. Kun ${ }^{1}$, A. Moór ${ }^{1}$, A. Farkas ${ }^{1}$, G. Hajdu ${ }^{7,8}$, G. Hodosán ${ }^{9}$, T. Kovács ${ }^{1}$, L. Kriskovics ${ }^{1}$, G. Marton ${ }^{1}$, L. Molnár ${ }^{1}$, \\ A. Pál ${ }^{1,10}$, K. Sárneczky ${ }^{1}$, Á. Sódor ${ }^{1}$, R. Szakáts ${ }^{1}$, T. Szalai ${ }^{11}$, E. Szegedi-Elek ${ }^{1}$, \\ A. Szing ${ }^{12}$, I. Tóth ${ }^{1}$, K. Vida ${ }^{1}$, and J. Vinkó ${ }^{1,11}$ \\ 1 Konkoly Observatory, Research Centre for Astronomy and Earth Sciences, Hungarian Academy of Sciences, PO Box 67, \\ 1525 Budapest, Hungary \\ e-mail: kospal@konkoly.hu \\ 2 Instituto de Astrofísica de Canarias, Avenida Vía Láctea, 38205 La Laguna, Tenerife, Spain \\ 3 Departamento de Astrofísica, Universidad de La Laguna, 38205 La Laguna, Tenerife, Spain \\ ${ }^{4}$ Harvard-Smithsonian Center for Astrophysics, 60 Garden Street, MS 78, Cambridge, MA 02138, USA \\ 5 Grantecan S. A., Centro de Astrofísica de La Palma, Cuesta de San José, 38712 Breña Baja, La Palma, Spain \\ ${ }^{6}$ Leiden Observatory, Leiden University, Post Office Box 9513, 2300 RA Leiden, The Netherlands \\ 7 Instituto de Astrofísica, Facultad de Física, Pontificia Universidad Católica de Chile, Av. Vicuña Mackenna, 4860 Santiago, Chile \\ 8 Instituto Milenio de Astrofísica, Santiago, Chile \\ 9 University of St Andrews, School of Physics \& Astronomy, St. Andrews, UK \\ 10 Department of Astronomy, Loránd Eötvös University, Pázmány Péter sétány 1/A, 1117 Budapest, Hungary \\ 11 Department of Optics and Quantum Electronics, University of Szeged, Dóm tér 9., 6720 Szeged, Hungary \\ 12 Baja Observatory, University of Szeged, 6500 Baja, KT. 766, Hungary
}

Received 30 December 2015 / Accepted 11 July 2016

\begin{abstract}
Context. HBC 722 (V2493 Cyg) is a young eruptive star in outburst since 2010. Spectroscopic evidence suggests that the source is an FU Orionis-type object, with an atypically low outburst luminosity.

Aims. Because it was well characterized in the pre-outburst phase, HBC 722 is one of the few FUors from which we can learn about the physical changes and processes associated with the eruption, including the role of the circumstellar environment.

Methods. We monitored the source in the BVRIJHK $K_{\mathrm{S}}$ bands from the ground and at 3.6 and $4.5 \mu \mathrm{m}$ from space with the Spitzer Space Telescope. We analyzed the light curves and studied the evolving spectral energy distribution by fitting a series of steady accretion disk models at many epochs covering the outburst. We also analyzed the spectral properties of the source based on our new optical and infrared spectra, comparing our line inventory with those published in the literature for other epochs. We also mapped HBC 722 and its surroundings at millimeter wavelengths.

Results. From the light-curve analysis we conclude that the first peak of the outburst in 2010 September was mainly due to an abrupt increase in the accretion rate in the innermost part of the system. This was followed after a few months by a long-term process, when the brightening of the source was mainly due to a gradual increase in the accretion rate and the emitting area. Our new observations show that the source is currently in a constant plateau phase. We found that the optical spectrum was similar in the first peak and following periods, but around the peak the continuum was bluer and the $\mathrm{H} \alpha$ profile changed significantly between 2012 and 2013. The source was not detected in the millimeter continuum, but we discovered a flattened molecular gas structure with a diameter of 1700 au and mass of $0.3 M_{\odot}$ centered on HBC 722 .

Conclusions. While the first brightness peak might be interpreted as a rapid fall of piled-up material from the inner disk onto the star, the later monotonic flux rise suggests the outward expansion of a hot component according to a previously described theory. Our study of HBC 722 demonstrates that accretion-related outbursts can occur in young stellar objects even with very low-mass disks in the late Class II phase.
\end{abstract}

Key words. stars: formation - circumstellar matter - infrared: stars - stars: individual: HBC 722

\section{Introduction}

Sun-like pre-main sequence stars are surrounded by circumstellar disks from which material is accreted onto the growing protostar. The accretion rate is variable: the protostar's normal

* This work is based on observations made with the Spitzer Space Telescope. Spitzer is operated by the Jet Propulsion Laboratory, California Institute of Technology under a contract with NASA. accretion at a low rate may be occasionally interspersed by brief episodes of highly enhanced accretion (Kenyon et al. 1990). FU Orionis-type variables (FUors) are thought to be the visible examples of episodic accretion. During their episodic outbursts, the accretion rate from the circumstellar disk onto the star increases by several orders of magnitude, from typically $10^{-7}$ up to $10^{-4} M_{\odot} \mathrm{yr}^{-1}$ (Audard et al. 2014). Owing to the increased accretion, FUors brighten by 5-6 mag at optical wavelengths, their 


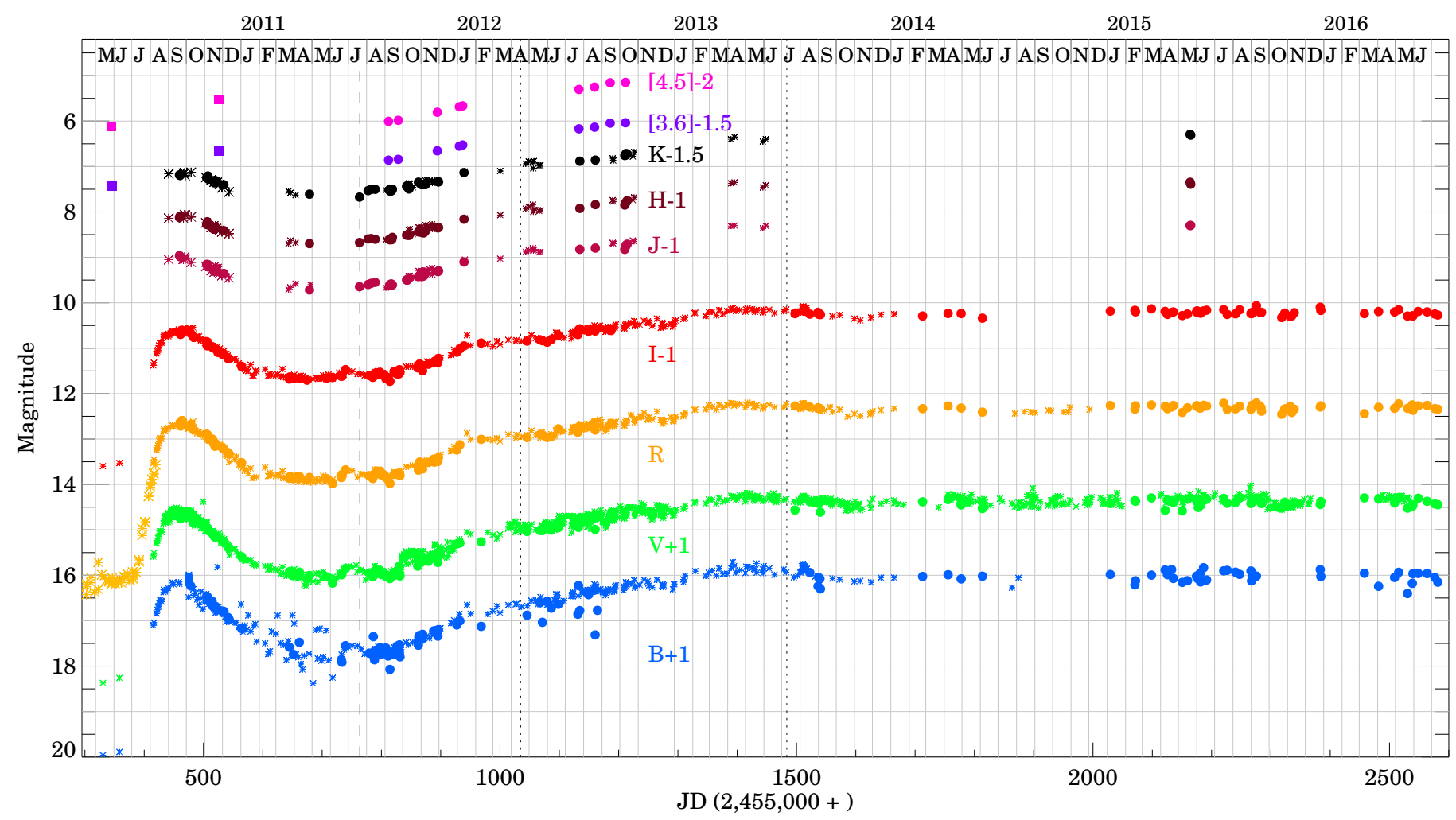

Fig. 1. Light curves of HBC 722. Filled dots are from Paper I and this work, plus signs are from Semkov et al. (2010), Semkov et al. (2014), Miller et al. (2011), Sung et al. (2013), Antoniucci et al. (2013), and from the AAVSO database ${ }^{1}$. Mid-IR data points are either from WISE (filled squares) or from Spitzer (filled dots). For clarity, the $B, V, I, J, H, K_{\mathrm{S}}$, [3.6] and [4.5] light curves are shifted along the $y$-axis. The vertical dashed line marks the epoch when our near-IR LIRIS spectum was taken, while the vertical dotted lines indicate the time of our optical GTC/OSIRIS spectra.

bolometric luminosities reach several hundred $L_{\odot}$, and they stay in the high state for several decades.

Currently, about two dozen FUors and FUor candidates are known. One of the recent discoveries, HBC 722 went into eruption in 2010 (Semkov \& Peneva 2010). The optical and near-infrared (near-IR) spectra of the object published by Miller et al. (2011) and Semkov et al. (2012) are similar to those of FU Ori and other FUors, confirming its FUor-type classification. HBC 722, however, differs from typical FUors in that its outburst luminosity and accretion rate are only on the order of $L_{\text {bol }}=10-20 L_{\odot}, \dot{M}=10^{-6} M_{\odot} \mathrm{yr}^{-1}$, well below what is typical of FUors (Kóspál et al. 2011, hereafter, Paper I). Despite this, HBC 722 has been in the bright state for at least five years now, it is currently brighter than ever (Baek et al. 2015), and it is on its way to exhibit a typical, decades-long FUor-type light curve.

HBC 722 is not an isolated object but a part of the $\mathrm{LkH} \alpha$ 188 cluster, a group of optically visible young stars showing $\mathrm{H} \alpha$ emission (Cohen \& Kuhi 1979). Several Class 0/I embedded protostars, as well as very young, very low-luminosity protostars or starless cores in the vicinity of HBC 722 indicate active star formation in the area (Green et al. 2011; Dunham et al. 2012). HBC 722 itself is a Class II object with a circumstellar disk (Miller et al. 2011; Kóspál et al. 2011), although with a rather low upper limit for the disk mass (Dunham et al. 2012). The system is surrounded by a reflection nebula (Miller et al. 2011).

HBC 722 is unique among FUors in that the progenitor, that is, the object in quiescence, has been well characterized. To learn about the physical changes and processes associated with the outburst, we carried out new optical and infrared photometric

\footnotetext{
1 http://www . aavso.org
}

monitoring of HBC 722, including mid-IR observations, which provide new information on the thermal emission of the inner disk at various phases of the outburst. We also obtained optical and near-IR spectra, as well as millimeter continuum and molecular line maps of the environment of the source, and compared our results with the FUor outburst theory of Bell \& Lin (1994).

\section{Observations and data reduction}

\subsection{Optical and infrared observations}

We obtained optical and near-IR images with BVRIJHK $K_{\mathrm{S}}$ filters between 2010 September 19 and 2016 July 12 using four telescopes: the Schmidt and RCC telescopes of the Konkoly Observatory (Hungary), and the IAC- 80 and TCS telescopes of the Teide Observatory on the Canary Islands (Spain). Technical details of the telescopes and their instrumentation are described in Paper I. Reduction of the images and aperture photometry was performed in the same way as in Paper I. The resulting magnitudes for the period between 2010 September 19 and 2011 January 2 are presented in Paper I, while the rest are listed in Table A.1. The light curves are plotted in Fig. 1.

We observed HBC 722 using the Spitzer Space Telescope in the post-helium phase at nine epochs between 2011 September 8 and 2012 October 12 (PID: 80165, PI: P. Ábrahám). We used the IRAC instrument at 3.6 and $4.5 \mu \mathrm{m}$ in full-array mode with exposure times of $0.2 \mathrm{~s}$ per frame. The data reduction and photometry was made in the same way as described in detail in Kun et al. (2011), except that here we used an aperture of 2 pixels (2".4) and sky annulus between 2-6 pixels (2'!4-7'!2). Convolution of the IRAC filter profiles with the observed spectral energy 
distribution (SED) proved that color correction is negligible. The results of the photometry are listed in Table A.2.

We observed HBC 722 with the LIRIS instrument installed on the $4.2 \mathrm{~m}$ William Herschel Telescope at the Observatorio del Roque de Los Muchachos (Spain). The description of the instrument can be found in Acosta-Pulido et al. (2007). JHK images and long-slit intermediate resolution spectra in the $Z J$ and $H K$ bands were obtained on 2011 July 20/21. The images were taken in a five-point dither pattern, with a $5 \mathrm{~s}$ exposure time per dither position. The spectra were taken in an ABBA nodding pattern with a total exposure time of $100 \mathrm{~s}$ and $60 \mathrm{~s}$ in the $Z J$ and $H K$ bands, respectively. We used the 0 '.75 slit width, which yielded a spectral resolution of $R=550-700$ in the $0.9-2.4 \mu \mathrm{m}$ range. We observed HIP 103694, a G2-type star, as telluric calibrator. The data of the images and spectra were reduced in the same way as in Acosta-Pulido et al. (2007). The spectra were flux calibrated using the $J H K_{\mathrm{S}}$ photometry taken on the same night. The typical signal-to-noise ratio $(\mathrm{S} / \mathrm{N})$ of the spectra is between 10 and 30. The photometry is included in Table A.1, while the spectra are plotted in Fig. 4.

HBC 722 was observed by the Wide-field Infrared Survey Explorer (WISE; Wright et al. 2010) in the cryogenic phase on 2010 May 28, and in the framework of the NeoWISE program on 2010 November 25. For both epochs, we downloaded all time-resolved observations from the AllWISE multiepoch photometry table in the $W 1(3.4 \mu \mathrm{m})$ and $W 2(4.6 \mu \mathrm{m})$ photometric bands and computed their average after removing outlier data points. Since the beam sizes of WISE in the two selected bands are approximately $6^{\prime \prime}$, no contamination from neighboring point sources is expected. We converted the computed magnitudes into fluxes and checked that similarly to Spitzer, color-correction was unnecessary. In the errors, we added in quadrature $2.4 \%$ and $2.8 \%$ as the uncertainty of the absolute calibration in the $W 1$ and W2 bands, respectively (Sect. 4.4 of the WISE Explanatory Supplement). The resulting fluxes are listed in Table A.2.

We carried out low-resolution spectroscopy of HBC 722 with the Optical System for Imaging and Low Resolution Integrated Spectroscopy (OSIRIS) tunable imager and spectrograph (Cepa et al. 2003; Cepa 2010) at the $10.4 \mathrm{~m}$ Gran Telescopio Canarias (GTC), located at the Observatorio Roque de los Muchachos in La Palma, Canary Islands, Spain, on 2012 April 17 and on 2013 July 10. Observations were performed in service mode within the GTC filler programs GTC55/12A and GTC3/13A. The heart of OSIRIS is a mosaic of two $4 \mathrm{k} \times 2 \mathrm{k}$ e2v CCD4482 detectors that gives an unvignetted field of view of $7.8 \times$ $7.8 \mathrm{arcmin}^{2}$ with a plate scale of $0.127 \mathrm{arcsec}_{\mathrm{pixel}}^{-1}$. However, to increase the $\mathrm{S} / \mathrm{N}$ of our observations, we chose the standard operation mode of the instrument, which is a $2 \times 2$-binning mode with a readout speed of $200 \mathrm{kHz}$. All spectra were obtained with the OSIRIS R2500R (red) grisms, covering the 5575-7685 wavelength range. Because of the highly variable seeing, we used the $1^{\prime \prime}$.23 slit oriented at the parallactic angle to minimize losses that are due to atmospheric dispersion, providing a dispersion of $1.6 \AA /$ pixel. The resulting wavelength resolution, measured on arc lines, was $R=2475$. The exposure time was $60 \mathrm{~s}$ in 2012 and $90 \mathrm{~s}$ in 2013. The spectra were reduced and analyzed using standard IRAF routines.

\subsection{Millimeter observations}

We observed HBC 722 at millimeter wavelengths with the Plateau de Bure Interferometer (PdBI) and with the IRAM $30 \mathrm{~m}$ telescope. The PdBI observations were taken on two different nights, 2012 March 28 and 2012 April 2. The PdBI antennas were in $6 \mathrm{Cq}$ configuration, providing a range of $u v$ radii between about $15 \mathrm{~m}$ and $175 \mathrm{~m}$. The total on-source correlation time was two hours. The weather conditions were good, with precipitable water vapor between 2 and $8 \mathrm{~mm}$ on March 28 and between 2 and $4 \mathrm{~mm}$ on April 2. We used the $3 \mathrm{~mm}$ receiver, tuned it halfway between the ${ }^{13} \mathrm{CO}$ and $\mathrm{C}^{18} \mathrm{O} J=1-0$ lines $(109.0918 \mathrm{GHz})$, and placed a $20 \mathrm{MHz}$-wide $39 \mathrm{kHz}$-resolution correlator unit on each CO line. We also used two $160 \mathrm{MHz}$ wide units to measure the $2.7 \mathrm{~mm}$ continuum emission. At this wavelength, the single-dish HPBW is 45".8. Bright quasars (3C 279, $2013+370$, and $\mathbf{J} 2120+445$ ) were observed at regular intervals to enable RF bandpass, phase, and amplitude calibration. The absolute flux scale was fixed using the flux standard carbon star MWC 349. The data were reduced in the standard way with CLIC, a GILDAS-based application written especially for reducing PdBI data. The rms phase noise was typically below $30^{\circ}$. We estimate a flux calibration accuracy of about $15 \%$.

The single-dish IRAM $30 \mathrm{~m}$ observations were taken during three nights between 2012 June 19 and 22, with precipitable water vapor between 5 and $9 \mathrm{~mm}$ and stable weather conditions. We obtained Nyquist-sampled $2^{\prime} \times 2^{\prime}$ on-the-fly (OTF) maps using the EMIR receiver in frequency-switching mode in the $110 \mathrm{GHz}$ band (HPBW was 22"). The map spacing was 7" with 17 OTF subscans. One map was obtained in about $7 \mathrm{~min}$, and this script was repeated several times to reach the desired sensitivity, in $6.25 \mathrm{~h}$. EMIR was used with two backends simultaneously. The Versatile SPectrometer Array (VESPA) provided $20 \mathrm{kHz}$ resolution and $60 \mathrm{MHz}$ bandwidth, which we used to observe the ${ }^{13} \mathrm{CO}$ and $\mathrm{C}^{18} \mathrm{O} J=1-0$ lines, to serve as short spacings for the PdBI data. The Fast Fourier Transform Spectrometer (FTS) was set in fine resolution mode, which we used to cover a wide bandwidth of $4 \times 1.8 \mathrm{GHz}$ between $93 \mathrm{GHz}$ and $114 \mathrm{GHz}$ with a resolution of $50 \mathrm{kHz}$, to search for additional lines in the spectrum. The resulting maps have an rms noise of $0.04-0.05 \mathrm{~K}$.

\section{Results and analysis}

\subsection{Light curves}

Figure 1 displays the multiband light curves of HBC 722, compiled from our observations and literature data. In addition to optical and near-IR measurements, our data set includes the first dedicated mid-infrared (3.6 and $4.5 \mu \mathrm{m})$ monitoring of a oneyear-long period of the outburst. The light curves show that the rapid brightening of HBC 722 started in 2010 mid-July and peaked in 2010 September in all bands between $B$ and $K$ (Miller et al. 2011; Semkov et al. 2014). The maximum was followed by a slower decay that lasted for about five months. Then the source stayed constant for another five months and again started to brighten. This monotonic flux rise was also clearly observed in the Spitzer data points at 3.6 and $4.5 \mu \mathrm{m}$. The increasing trend has a turning point in 2012 January, when the brightening slowed down. The fluxes at all wavelengths reached a plateau in 2013 April, and our recent photometry from 2015-2016 (Table A.1) demonstrates that they have been approximately constant since then.

Optical monitoring of the source before the outburst (Miller et al. 2011; Semkov et al. 2012) revealed that what appears as the starting point of the outburst in Fig. 1 in 2010 May is already an elevated flux level compared to the brightness of the source before 2010. Thus, similarly to Miller et al. (2011), we consider the 2010 May state as part of the outburst, as an 

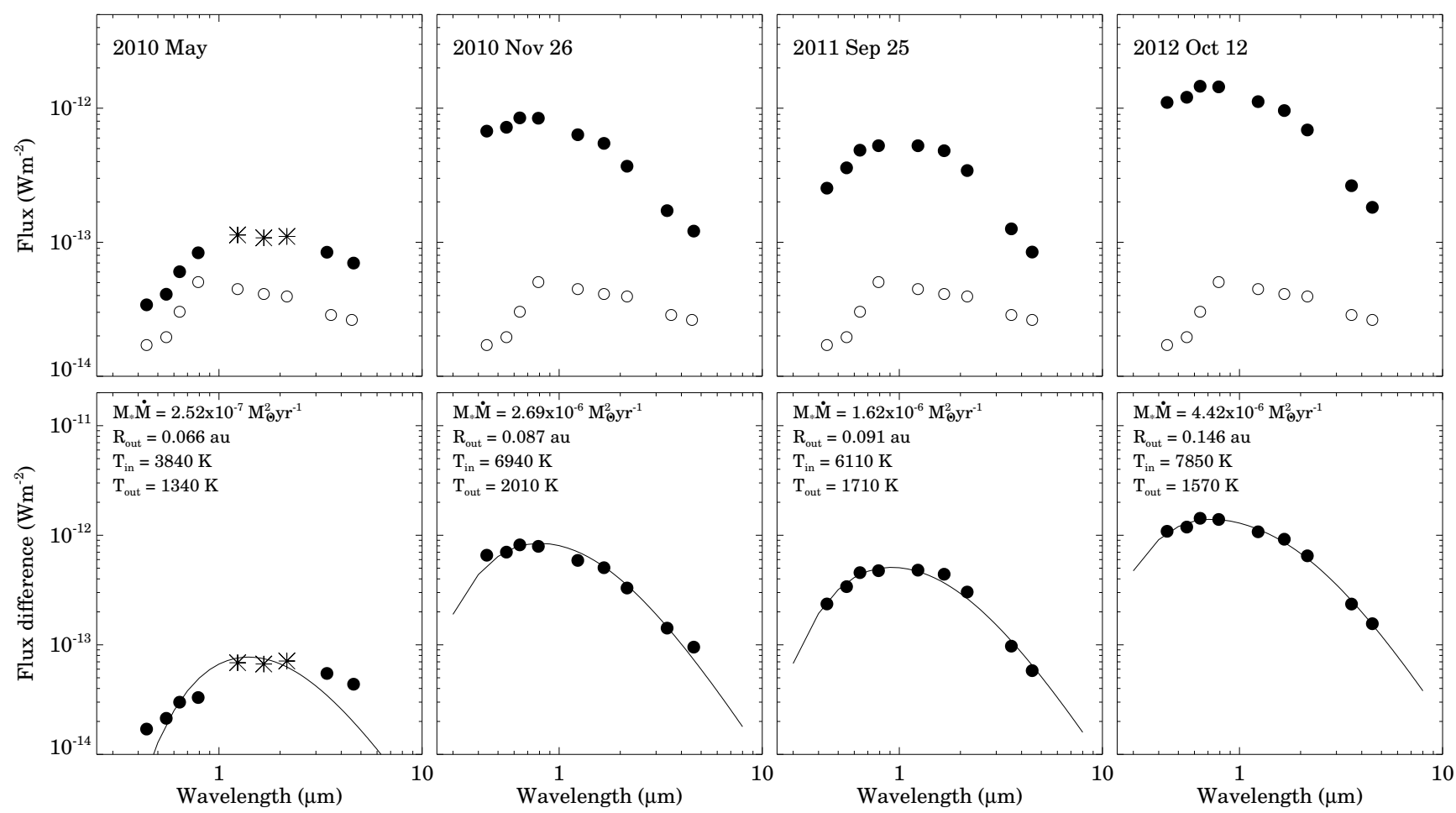

Fig. 2. SEDs of HBC 722 at four different representative epochs of the outburst. The panels in the upper row present the actual SED (filled symbols), with the quiescent SED from 2006 (open symbols) overplotted. The first epoch in 2010 May corresponds to the kickoff of the outburst just preceding the rapid first brightening, when the source was already in an elevated state compared to the quiescent one. In 2010 May the $J H K_{\mathrm{S}}$ data points (asterisks) are extrapolations from later observations. The lower panels show the difference flux between the actual and the quiescent SEDs for the four epochs, as well as accretion disk fits to the difference.

initial pedestal where the rapid brightening started from (hereafter called kickoff state).

While the general shapes of the light curves in different bands are similar, the amplitudes of the light variations in Fig. 1 are not the same. This wavelength dependence carries information on the physical processes responsible for the flux changes. We constructed an SED for each night for which near- or midIR observations were available. If simultaneous optical data (obtained on the same night) were not available, we interpolated in the BVRI light curves. We created separate SEDs for the kickoff state (2010 May) and for the true quiescent state (before 2010, not plotted in Fig. 1) of the system. For the latter, we collected photometric data obtained between 2006 March and October, namely optical measurements from Semkov et al. (2012), UKIDSS $J H K_{\mathrm{S}}$ from Paper I, and Spitzer 3.6 and $4.5 \mu \mathrm{m}$ data points from Rebull et al. (2011). A comparison between the quiescent and the kickoff SEDs is shown in Fig. 2 (upper left panel).

The optical and near- to mid-infrared excesses arise from the innermost part of the circumstellar disk, which we modeled with an optically thick, viscous accretion disk of radially constant mass accretion rate. Our approach is similar to what Zhu et al. (2007) adopted for FU Ori. The main difference is that we aim to reproduce only broad-band photometry and not line spectroscopy, therefore a vertical treatment of the disk atmosphere is not necessary. At each epoch we assumed a steady, geometrically thin, optically thick disk, whose radial temperature profile is (Hartmann \& Kenyon 1996):

$T_{\mathrm{d}}^{4}(R)=\frac{3 G M \dot{M}}{8 \pi R^{3} \sigma}\left[1-\left(\frac{R_{*}}{R}\right)^{1 / 2}\right]$, where $T_{\mathrm{d}}$ is the disk temperature at radius $R, M$ is the stellar mass, $\dot{M}$ is the accretion rate, $R_{*}$ is the stellar radius, $G$ is the gravitational constant, and $\sigma$ is the Stefan-Boltzmann constant. To avoid the unphysical zero temperature at $R=R_{*}$, we followed Zhu et al. (2007) to prescribe $T=T_{\max }$ within $1.36 R_{*}$, the radius of maximum temperature. Then we calculated the disk SED by integrating the fluxes of concentric annuli between $R_{*}$ and $R_{\text {out }}$. The annuli were assumed to emit blackbody radiation corresponding to the temperature defined by Eq. (1) at a distance of $550 \mathrm{pc}$. The observed variations of the light curves can then be interpreted in terms of temporal changes in two parameters: $M \dot{M}$ and $R_{\text {out }}$.

We converted the observed magnitudes into fluxes using the appropriate flux values for zero magnitudes from the literature. Since we intended to analyze the extra brightening related to the outburst, we subtracted the quiescent SED, which represents the sum of the photosphere of the central star and the quiescent emission of the (passive) circumstellar disk, from each epoch. Examples for four representative epochs are shown in Fig. 2. Then, we corrected for interstellar reddening by adopting $A_{V}=3.1 \mathrm{mag}$ (Miller et al. 2011). We fit our accretion disk model to the resulting SEDs. At each epoch we used all available data points, except the kickoff state (2010 May), when the $\lambda \geq 3.5 \mu \mathrm{m}$ section of the SED markedly deviated from our accretion disk model and was excluded from the fit. Accidentally low individual error bars would result in additional weight on certain data points in the fitting routine, which would influence the fit result. Thus, we set a minimum formal uncertainty value of $3 \%$ for the fitting procedure, and all error bars below this threshold were increased to $3 \%$.

For the stellar radius we adopted $R_{*}=1.51 R_{\odot}$, computed from a stellar luminosity of $L_{*}=0.67 L_{\odot}$, corresponding to a 


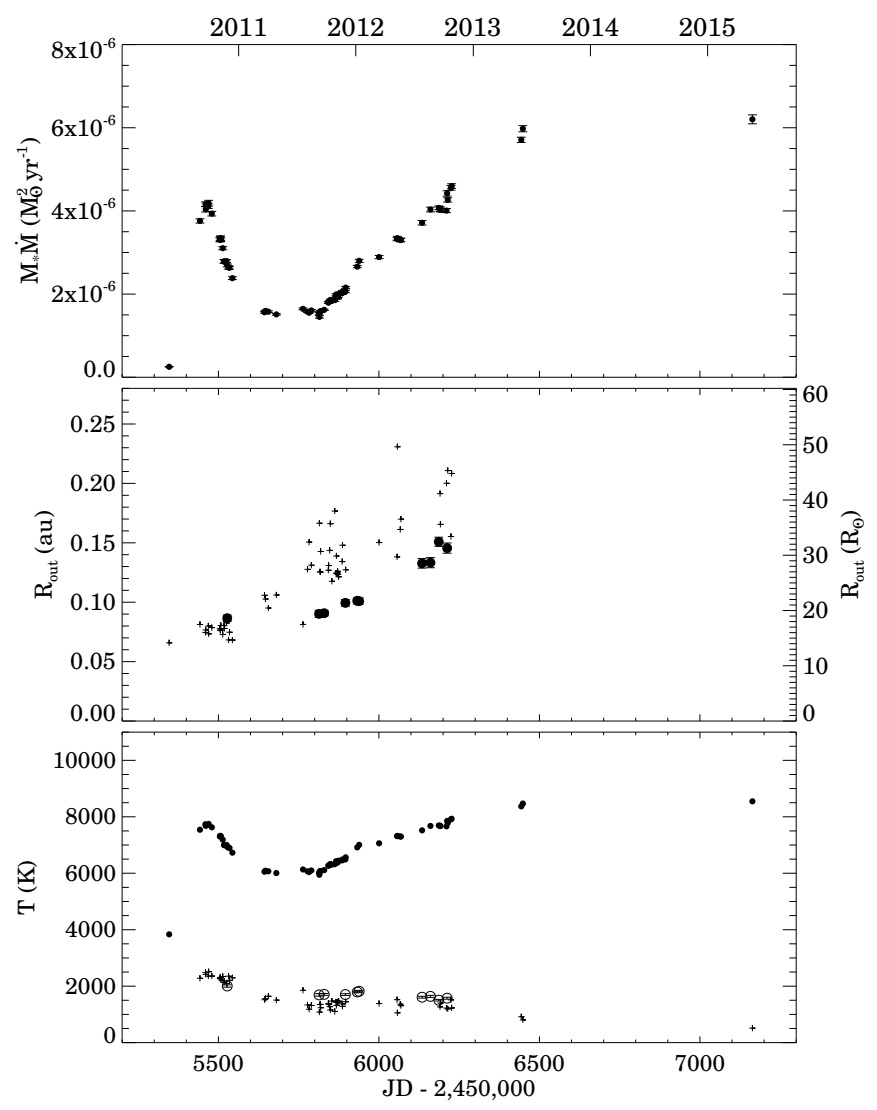

Fig. 3. Time evolution of the accretion rate (multiplied by the stellar mass), the outer radius, and the temperature at the inner and outer edges of the accretion disk. In the middle panel the black dots mark the epochs when $\lambda \geq 3.4 \mu \mathrm{m}$ data points are available for the fitting.

Kurucz stellar photosphere with $T_{\text {eff }}=4250 \mathrm{~K}$ at a distance of $550 \mathrm{pc}$ (Castelli \& Kurucz 2004). This model atmosphere provided the best match to our BVRIJH data points in the quiescent phase. The accretion disk model resulted in reasonable fits to the SEDs, but reproducing the shortest wavelength data points in the $B$ - and $V$-bands turned out to be sensitive to disk inclination. A closer to edge-on geometry would correspond to a smaller projected disk area, which requires a higher temperature, that is, a higher accretion rate, to match the observed level of optical-infrared emission. A too high temperature, however, would produce too much short-wavelength radiation, thus fitting the $B$ - and $V$-bands offers a possibility to constrain the inclination. Minimizing a total $\chi^{2}$, computed by adding the $\chi^{2}$ values of the individual epochs for the whole outburst, yielded an inclination value of $73_{-15}^{+6} \mathrm{deg}$, which means that the system is seen in close to edge-on.

Adopting this inclination value, we obtained good fits for all SEDs in our monitoring program. In Fig. 2 we overplot the bestfit results at the four representative epochs. This demonstrates that the excess emission related to the outburst is fully consistent with an accretion disk profile. The time evolution of the fitted parameters and derived temperature at the inner and outer edges of the disk are plotted in Fig. 3. We note that the outer radius is better constrained when mid-IR data points are available (black dots), while it has a higher uncertainty at those epochs when $K$-band was the longest wavelength included in the fit. Nevertheless, all data points outline similar general trends.

Our simple accretion disk models indicate $M \dot{M}$ values of a few times $10^{-6} M_{\odot}^{2} \mathrm{yr}^{-1}$. Assuming a stellar mass of $0.8-0.9 M_{\odot}$, typical for young K7-type stars (e.g., Siess et al. 2000), the resulting accretion rates are somewhat higher than published earlier for HBC 722, calculated from the accretion luminosity $\left(\sim 10^{-6} M_{\odot} \mathrm{yr}^{-1}\right.$, Kóspál et al. 2011; Green et al. 2013b). The difference is mainly related to the flat-disk geometry and the more edge-on than face-on inclination. Earlier results from the literature support the relatively high accretion rate and close to edge-on geometry. Green et al. (2013a) observed exceptionally strong [OI] line emission at $63.18 \mu \mathrm{m}$, suggesting stronger accretion than what is calculated from the accretion luminosity. Our inclination angle is close to the value of $85^{\circ}$ suggested by Gramajo et al. (2014) from SED modeling. The rapid change in the line-of-sight extinction, derived from multiepoch X-ray observations by Liebhart et al. (2014), may also point to a more edge-on than pole-on configuration.

The uncertainties associated with the disk inclination, stellar mass, distance, and especially the applicability of our simple disk model make the absolute value of the accretion rate uncertain. As a test, we repeated the fitting process by relaxing the assumption in Eq. (1) that the inner edge of the disk is equal to the stellar radius, and let it be a free parameter in the fitting process. While a trend for slightly increasing inner radius with time was shown by the best fits, this model family requires an even closer to edge-on geometry $\left(\geq 82^{\circ}\right)$ and $2-4$ times higher accretion rates, suggesting that the derived absolute values of $M \dot{M}$ should be used with caution. However, the temporal trends seen in the accretion rate and outer radius in Fig. 3 are very reliable, and we analyze these trends in the following.

Figure 3 reveals that the first peak of the outburst in 2010 September was caused by a rapid increase in the accretion rate. This corresponded to a rise in the inner disk temperature. The peak was followed by a large drop in the accretion rate and a somewhat smaller drop in the temperature, which explains the fading of the source between 2010 September and 2011 February. During this fading, the outer radius of the accretion disk stayed constant at $0.08-0.09 \mathrm{au}$, with an outer disk temperature above $2000 \mathrm{~K}$. Next, from 2011 autumn, the accretion rate started a gradual increase, leading to higher maximum disk temperatures and causing the rebrightening of the source. The outer radius also increased, approximately linearly with time, while the temperature remained constant at $\sim 1500 \mathrm{~K}$, which is due to the combined effects of increasing accretion rate and increasing outer radius. The information on the further evolution after 2012 November is sporadic and less conclusive because there are only three epochs with near-IR data and no mid-IR measurements. The derived accretion rate seems to increase slightly further until mid-2013, then remains constant. The fitted outer disk radii at these three epochs are unexpectedly large $(0.34 \mathrm{au}, 0.41 \mathrm{au}$, and $0.77 \mathrm{au}$; not plotted in Fig. 3), but also very uncertain. Nevertheless, the formal outer disk temperatures at these three epochs became low, below $1000 \mathrm{~K}$.

Our results suggest that the outburst of HBC 722 was driven by two separate physical processes. The main one is the linear rise in the accretion rate and the outer radius of the accretion disk that was dominant from 2011 July until the plateau phase. Superimposed on this is another process that caused the first brightness peak, dominated the outburst evolution between 2010 August and 2011 February, and disappeared afterwards.

\subsection{Optical and near-IR spectroscopy}

Our near-IR spectrum in Fig. 4 exhibits several spectral features, all in absorption. In the $Z J$ band, $\mathrm{Pa} \beta$ is the most conspicuous line, but other lines of the Paschen series from Pay 
to Pa9 are also tentatively detected. The drop of the spectrum toward longer wavelengths indicates the beginning of a broad water band. In the $H$ band, no secure detection of lines is possible. The general shape of the spectrum indicates another deep water absorption band starting at $1.7 \mu \mathrm{m}$. In the $K$ band, Ca lines and the $\operatorname{Br} \delta$ may be present. No detection of $\operatorname{Br} \gamma$ is evident. The $\mathrm{CO}$ band-head features are very well visible. Our spectrum was taken after the first brightness peak, but before the source started to rebrighten (2012 July). Lorenzetti et al. (2012) also obtained near-IR spectra around the same phase of the light curve (2012 August-September). Except for the different spectral resolutions, their spectra are similar to ours in the spectral features, general spectral shape, and absolute flux levels. All the features we detected have been present in the spectrum taken during the first peak (Miller et al. 2011; Lorenzetti et al. 2012). The shape of our spectrum indicates redder emission and more pronounced water absorption than at peak brightness. The color change is consistent with the temperature changes we concluded from our light-curve analysis (Fig. 3).

Our first optical spectrum was taken when HBC 722 was rebrightening. By the time we took the second optical spectrum, the light curve reached the plateau phase. The spectra indicate a gradually rising continuum with the $\mathrm{H} \alpha$ line displaying a complex profile, and several other lines in absorption, including the Na D lines ( $5892 \AA, 5898 \AA$ ), two Ba II lines (6499 and the Li I line (6709 $\AA$ ). These lines are the same as those identified by Miller et al. (2011) in the corresponding wavelength range for a spectrum taken close to the first brightness peak. Our two spectra are remarkably similar, almost indistinguishable from each other, except for the Na D lines, which became weaker, and for the $\mathrm{H} \alpha$ line, whose profile changed from 2012 to 2013 (Fig. 4). The $\mathrm{H} \alpha$ line in the spectra of Miller et al. (2011) showed a pure emission feature. The monitoring of Lee et al. (2015) revealed a time evolution of the $\mathrm{H} \alpha$ feature. This developed a PCygni profile already at the beginning of the outburst, which was attributed to a wind component. Our spectrum from 2012 April also shows a clear P Cygni profile. This is expected because a slowly evolving $\mathrm{H} \alpha$ feature was observed for V1057 Cyg (Herbig 1977) and for V1647 Ori (Aspin \& Reipurth 2009). Interestingly, Lee et al. (2015) found that the wind component disappeared by the end of 2012, and then the line profile exhibited a central absorption with emission wings on both sides. Our measurement from 2013 July is consistent with this line shape.

\subsection{Millimeter images}

Interferometric continuum data. Figure 5 shows our $2.7 \mathrm{~mm}$ continuum image of the area around HBC 722. The target itself is not visible, and from the rms noise of the image, we deduce a $3 \sigma$ upper limit of $0.24 \mathrm{mJy}^{\text {beam }^{-1}}$ for its $2.7 \mathrm{~mm}$ flux. This corresponds to a point source upper limit for the total circumstellar mass of about $0.01 M_{\odot}$, using a gas-to-dust mass ratio of 100 . Dunham et al. (2012) mapped the area at $1.3 \mathrm{~mm}$ with the SMA using projected baselines between $5 \mathrm{~m}$ and $76 \mathrm{~m}(3.8-58.5 \mathrm{k} \lambda)$. These largely overlap with the projected baselines of our IRAM PdBI observations (between 15 and $175 \mathrm{~m}$ or $5.6-64.8 \mathrm{k} \lambda$ ), resulting in similar synthesized beams $\left(2^{\prime \prime} .73 \times 33^{\prime \prime} 02\right.$ at PA $-48^{\circ}$ for SMA and $2 ! \cdot 74 \times 2$ × 21 at PA $96^{\circ}$ for IRAM). This means that the two images trace the same spatial scales and can be safely compared. The comparison in Fig. 5 shows a remarkable similarity between the two images at different millimeter wavelengths. Dunham et al. (2012) did not detect HBC 722 either,
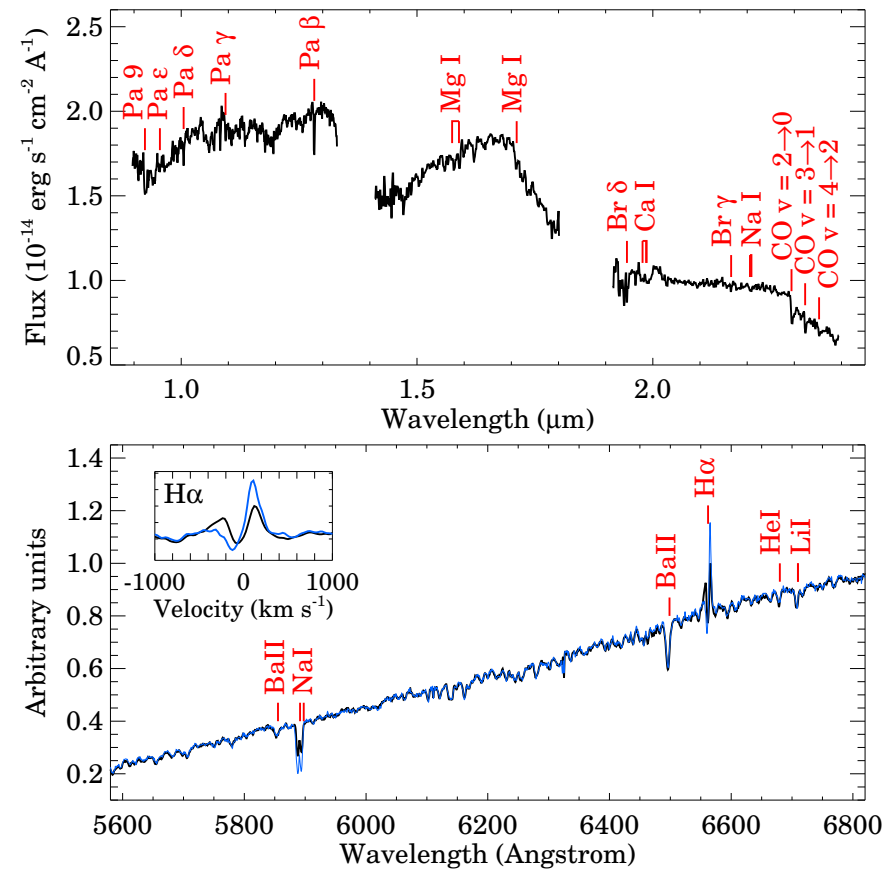

Fig. 4. Top panel: near-IR spectrum of HBC 722 obtained with WHT/LIRIS on 2011 July 21. To guide the eye, the vertical red bars show the laboratory wavelengths of atomic and molecular features typical for young stars. Bottom panel: optical spectra of HBC 722 obtained with GTC/OSIRIS on 2012 April 17 (blue curve) and 2013 July 10 (black curve). The inset shows the $\mathrm{H} \alpha$ line in more details as a function of the velocity. The identified lines are marked in red above the optical spectra.

their $3 \sigma$ upper limit was $5 \mathrm{mJy}_{\text {beam }}^{-1}$ at $1.3 \mathrm{~mm}$, giving a similar upper limit for the total mass as the value from our $2.7 \mathrm{~mm}$ measurement. They identified seven sources in the vicinity of HBC 722, named MMS1 to MMS7, marked in Fig. 5. These sources, claimed to be embedded protostars or starless cores by Dunham et al. (2012), may have formed together with HBC 722. With the exception of MMS7, we detected all these millimeter sources in our $2.7 \mathrm{~mm}$ map. By fitting 2D Gaussians, we determined their peak fluxes, total fluxes, deconvolved sizes, and position angles, which are listed in Table A.3. Our photometry, together with that in Dunham et al. (2012), enabled us to calculate the millimeter spectral slopes of the detected sources. For this, we plotted the SEDs of the sources $v F_{v}$ as a function of wavelength. For the three sources with the best signal-to-noise millimeter detection, we determined the slope $\alpha$ from the $1.3 \mathrm{~mm}$ and $2.7 \mathrm{~mm}$ points. For optically thin emission, $\alpha$ is related to the spectral index of the dust opacity, $\beta$, as follows: $\beta=\alpha-3$. For the ISM, $\beta$ is about 1.7. If there is significant grain growth, $\beta$ should be lower, typically between 0 and 1 (e.g., Ricci et al. 2010). We found that two sources, MMS3 and MMS4, have ISM-like dust $(\beta=1.6 \pm 0.3$ for MMS3 and $\beta=1.5 \pm 0.3$ for MMS4), while MMS1 shows signs of grain growth $(\beta=0.7 \pm 0.3)$.

Interferometric and single-dish CO line data. By examining the channel maps, we determined that significant $\mathrm{CO}$ emission comes from the $0-9 \mathrm{~km} \mathrm{~s}^{-1}$ velocity range. The left panel of Fig. 6 shows the total intensity of the ${ }^{13} \mathrm{CO}(1-0)$ emission integrated for this wide velocity range. There is a roughly circular clump with a diameter of about $50^{\prime \prime}$ with several brightness peaks throughout the image, one of which may be associated with MMS1, while the other compact mm sources are not 


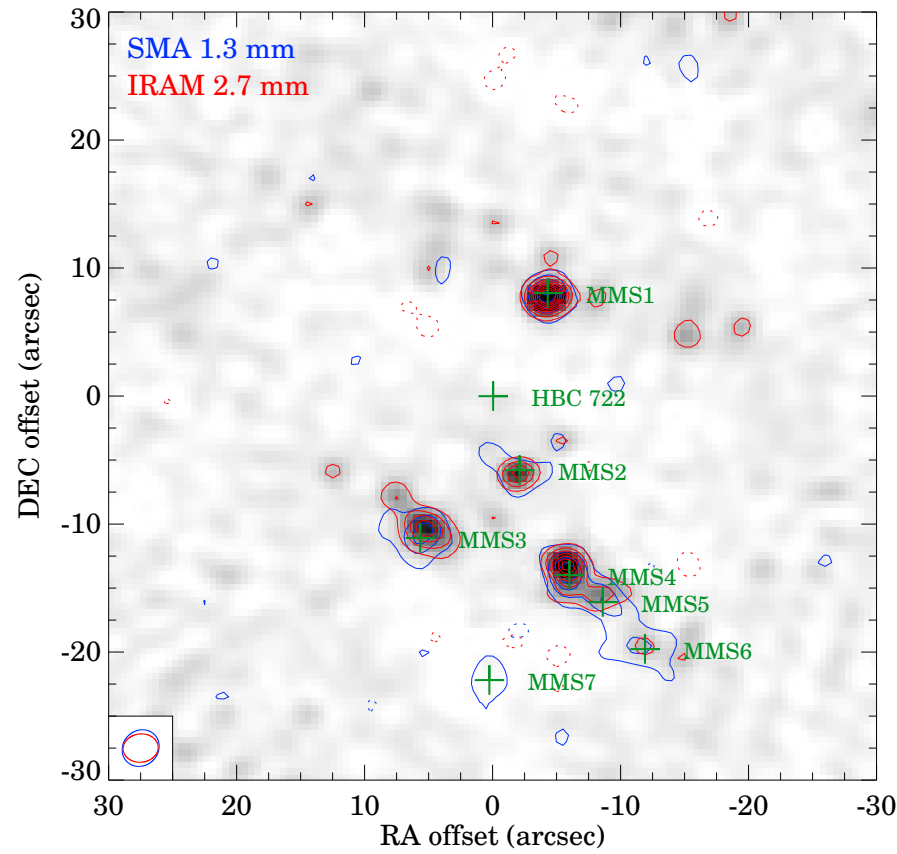

Fig. 5. Millimeter continuum emission map of the area around HBC 722. The grayscale image and the red contours are IRAM PdBI $2.7 \mathrm{~mm}$ observations from this work, while the blue contours are SMA $1.3 \mathrm{~mm}$ data from Dunham et al. (2012). At $2.7 \mathrm{~mm}$, contours are displayed at $4,8,12, \ldots \sigma$, with $\sigma=0.08 \mathrm{mJy}$, while at $1.3 \mathrm{~mm}$, contours are at $2.5,5,7.5, \ldots \sigma$, with $\sigma=1.65 \mathrm{mJy}$.

detected. Emission coinciding with the position of HBC 722 is detected in a limited velocity range, between 5.45 and $6.52 \mathrm{~km} \mathrm{~s}^{-1}$. The right panel of Fig. 6 shows the total intensity of the ${ }^{13} \mathrm{CO}(1-0)$ emission integrated for this narrow velocity range. The structure centered on HBC 722 is slightly elongated in the northwest-southeast direction with a deconvolved size of about $1700 \mathrm{au}$, while it remains unresolved in the perpendicular direction. The integrated flux of this source is $1.7 \mathrm{Jy} \mathrm{km} \mathrm{s}^{-1}$, corresponding to a total gas mass of $0.03 M_{\odot}$ assuming a temperature of $32 \mathrm{~K}$ (see below). The source is also visible in the $\mathrm{C}^{18} \mathrm{O}$ map integrated for the same narrow velocity range, and its flux of $0.22 \mathrm{Jy} \mathrm{km} \mathrm{s}^{-1}$ gives the same gas mass estimate, indicating that both lines are optically thin.

In the left panel of Fig. 6 we also indicate for reference the ${ }^{13} \mathrm{CO}(2-1)$ emission measured with SMA by Dunham et al. (2012) with contours, integrated for the wide $0-9 \mathrm{~km} \mathrm{~s}^{-1}$ velocity range. We note that the two images cannot be directly compared because our IRAM map combines the interferometric and single-dish data, that is, includes zero and short spacings, while the SMA map only contains interferometric measurements, which means that some extended flux is filtered out. Spatial filtering probably explains the significant differences between the IRAM and SMA maps. When we restricted the imaging to the PdBI data and did not include short spacings from the IRAM $30 \mathrm{~m}$ telescope, we obtained a similar map to that of Dunham et al. (2012). Assuming local thermodynamic equilibrium (LTE) and optically thin emission for the ${ }^{13} \mathrm{CO}$ isotopolog, the two interferometric-only maps can be used to estimate the temperature of the emitting gas according to the Maxwell-Boltzmann distribution. For this purpose we integrated the flux in an area of $10^{\prime} \times 10^{\prime}, 20^{\prime} \times 20^{\prime}$, and $40^{\prime} \times 40^{\prime}$ centered on HBC 722. We obtained temperatures of $32 \mathrm{~K}, 22 \mathrm{~K}$, and $20 \mathrm{~K}$, respectively, which means that there is an indication of a temperature gradient.
With IRAM we also obtained a map of the $\mathrm{C}^{18} \mathrm{O}(1-0)$ emission, which shows a spatial distribution very similar to that of ${ }^{13} \mathrm{CO}(1-0)$. The line ratio of the two isotopologs is around 8-9 throughout the map. The standard ${ }^{13} \mathrm{CO} / \mathrm{C}^{18} \mathrm{O}$ abundance ratio in the ISM is 8.1 (e.g., Wilson \& Rood 1994), therefore our measurements indicate that both $\mathrm{C}^{18} \mathrm{O}$ and ${ }^{13} \mathrm{CO}(1-0)$ are optically thin and can be used to estimate the total gas mass in the area. Using $22 \mathrm{~K}$ as a representative temperature and the canonical $\mathrm{H}_{2} /{ }^{12} \mathrm{CO}$ abundance ratio of $10^{4},{ }^{12} \mathrm{CO} /{ }^{13} \mathrm{CO}$ abundance ratio of 69 , and ${ }^{12} \mathrm{CO} / \mathrm{C}^{18} \mathrm{O}$ abundance ratio of 560 (Wilson \& Rood 1994), we obtained $7.6 M_{\odot}$ from the ${ }^{13} \mathrm{CO}$ flux and $7.3 M_{\odot}$ from the $\mathrm{C}^{18} \mathrm{O}$ flux. The calculated mass is between $6.9 M_{\odot}$ and $10.1 M_{\odot}$ for temperatures between $20 \mathrm{~K}$ and $32 \mathrm{~K}$.

We calculated first- and second-moment maps from our IRAM data cubes. We detected no significant velocity gradient in the area of HBC 722. The line profiles are fairly similar at all points of the maps with an average systemic velocity of $4.7 \pm 0.2 \mathrm{~km} \mathrm{~s}^{-1}$. The line profiles are fairly broad, the average line widths are $3.4 \pm 0.3 \mathrm{~km} \mathrm{~s}^{-1}$ for ${ }^{13} \mathrm{CO}$ and $3.1 \pm 0.4 \mathrm{~km} \mathrm{~s}^{-1}$ for $\mathrm{C}^{18} \mathrm{O}$.

The size of the $\mathrm{CO}$ emitting region in the left panel of Fig. 6 is roughly consistent with the extension of the optical reflection nebula visible around HBC 722 in outburst (Miller et al. 2011). However, while the CO emission is rather spherical, the optical nebula is highly asymmetric, being more extended toward the southwest, which indicates anisotropic illumination. Armond et al. (2011) observed that HBC 722 is surrounded by $\mathrm{H} \alpha$, [SII], and $\mathrm{H}_{2}$ knots within $10^{\prime \prime}$ of the star, which they called $\mathrm{HH}$ 655. There is no clear CO counterparts of these structures in our CO channel maps. We searched for outflow signatures as well, but detected none.

Single-dish line data. To search for emission from different molecules, we obtained a wide bandwidth spectrum with the FTS spectrograph on the IRAM 30 m telescope. We identified the following species and transitions (in order of increasing frequency):

- the CS $J=2-1$ line at $97.981 \mathrm{GHz}$

- the $\mathrm{HC}_{3} \mathrm{~N} J=12-11$ line at $109.174 \mathrm{GHz}$;

- the $\mathrm{C}^{18} \mathrm{O} J=1-0$ line at $109.782 \mathrm{GHz}$;

- the HNCO $J_{\mathrm{K}_{-1} \mathrm{~K}_{+1}}=5_{05}-4_{04}$ line at $109.906 \mathrm{GHz}$;

- the ${ }^{13} \mathrm{CO} J=1-0$ line at $110.201 \mathrm{GHz}$;

- the $\mathrm{C}^{17} \mathrm{O} J=1-0$ hyperfine triplet at $112.359 \mathrm{GHz}$; and

- several CN $N=1-0$ lines at 113.123, 113.144, $113.170,113.191,113.488,113.491,113.500,113.509$, and $113.520 \mathrm{GHz}$.

These are all low-excitation transitions with upper level energies of $5.3 \mathrm{~K}$ for ${ }^{13} \mathrm{CO}$ and $\mathrm{C}^{18} \mathrm{O}, 5.4 \mathrm{~K}$ for $\mathrm{C}^{17} \mathrm{O}$ and $\mathrm{CN}, 7.1 \mathrm{~K}$ for $\mathrm{CS}, 10.6 \mathrm{~K}$ for $\mathrm{HNCO}$, and $34.1 \mathrm{~K}$ for $\mathrm{HC}_{3} \mathrm{~N}$. The line profiles, obtained by integrating the data cubes for an area of $100^{\prime \prime} \times$ $100^{\prime \prime}$, are plotted in the right column of Fig. 7. The line profiles are approximately Gaussian, with FWHM between $0.6 \mathrm{~km} \mathrm{~s}^{-1}$ and $2.2 \mathrm{~km} \mathrm{~s}^{-1}\left({ }^{13} \mathrm{CO}\right.$ is the widest, HNCO is the narrowest), and the lines peak at a velocity between $4.6 \mathrm{~km} \mathrm{~s}^{-1}$ and $6.0 \mathrm{~km} \mathrm{~s}^{-1}$.

The left column of Fig. 7 displays the total line intensity maps integrated between $-2 \mathrm{~km} \mathrm{~s}^{-1}$ and $10 \mathrm{~km} \mathrm{~s}^{-1}$. The $\mathrm{CO}$ emission forms a large round clump, similar in all three isotopologs. Other lines are more compact, peaking in the triangle formed by HBC 722, MMS3, and MMS4. The CS emission is more elongated toward the west. The matching velocities, similar spatial locations, and low-excitation temperatures indicative of cold 

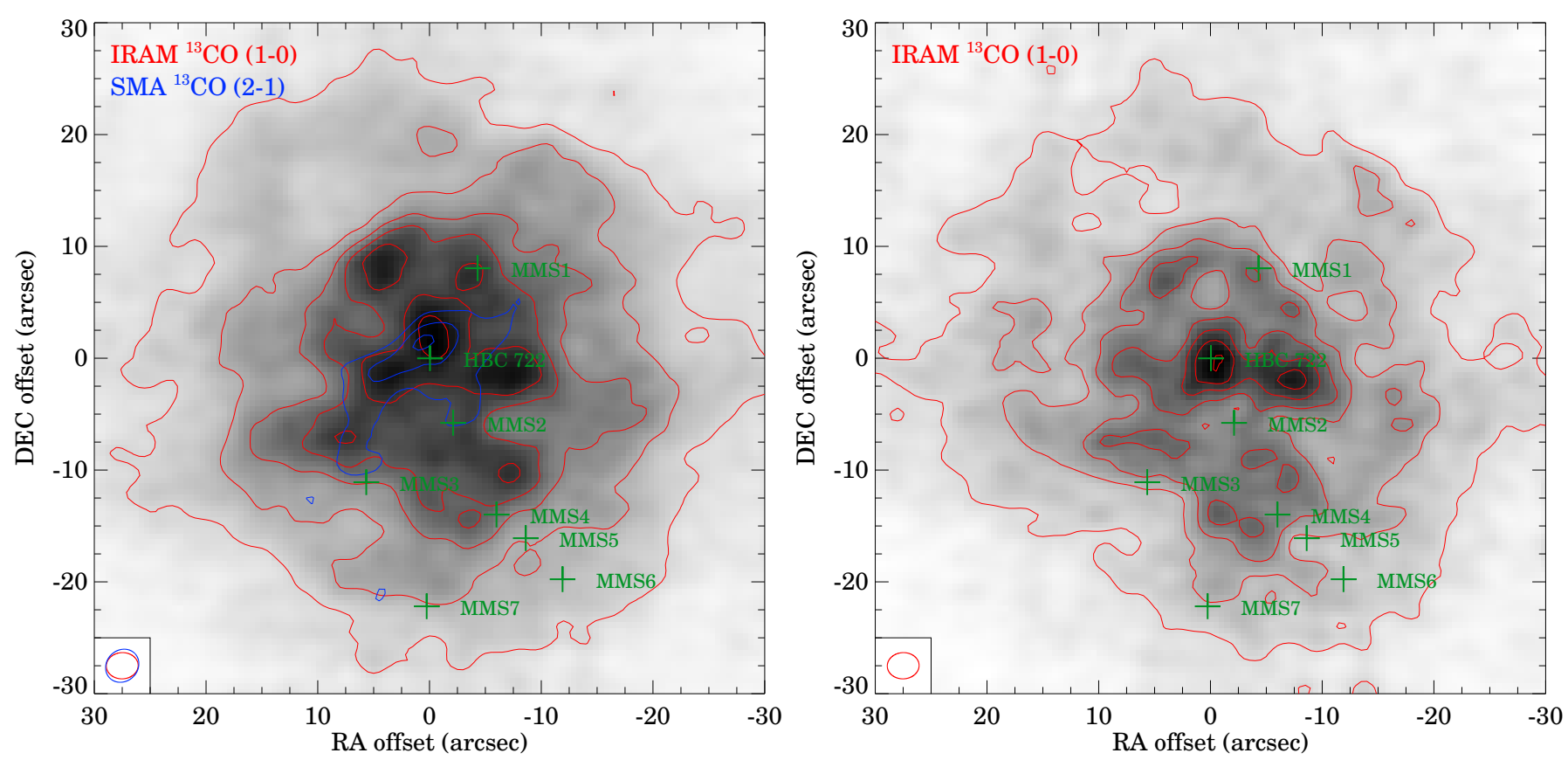

Fig. 6. Left: total intensity of the ${ }^{13} \mathrm{CO}$ emission in the area around $\mathrm{HBC} 722$, integrated for the $0-9 \mathrm{~km} \mathrm{~s}^{-1}$ velocity range. The grayscale image and the red contours are IRAM PdBI+30 m observations from this work, while the blue contours are SMA data from Dunham et al. (2012). For the ${ }^{13} \mathrm{CO}(1-0)$ line, contours are displayed at $4,8,12, \ldots \sigma$, with $\sigma=0.09 \mathrm{Jy} \mathrm{km} \mathrm{s}{ }^{-1}$, while for the ${ }^{13} \mathrm{CO}(2-1)$ line, contours are at $4,8,12, \ldots \sigma$, with $\sigma=0.3 \mathrm{Jy} \mathrm{km} \mathrm{s}^{-1}$. Right: total intensity of the ${ }^{13} \mathrm{CO}$ emission in the area around HBC 722, integrated for the $5.46-6.52 \mathrm{~km} \mathrm{~s}^{-1}$ velocity range. Contours are at $4,8,12, \ldots \sigma$, with $\sigma=0.03 \mathrm{Jy} \mathrm{km} \mathrm{s}^{-1}$.

gas suggest that all gas lines are probably coming from the cloud that formed the young stars and cores.

For comparison, we plot in Fig. 7 the Herschel/SPIRE $350 \mu \mathrm{m}$ image of the area around HBC 722. At this wavelength, Herschel's beam size is approximately the same as that of the IRAM $30 \mathrm{~m}$ telescope at $3 \mathrm{~mm}$. The spatial distribution of $350 \mu \mathrm{m}$ continuum emission is very similar to that of the $\mathrm{CN}$ and $\mathrm{HC}_{3} \mathrm{~N}$ lines, and to a lesser degree also to the CS and HNCO lines. It peaks around MMS3 and MMS4 and is relatively compact. The emission of the three $\mathrm{CO}$ isotopologs, on the other hand, is more extended. This is expected because the critical density is $2-3 \times 10^{3} \mathrm{~cm}^{-3}$ for $\mathrm{CO}$ and $4 \times 10^{5}-2 \times 10^{6} \mathrm{~cm}^{-3}$ for the other molecules, which are high-density gas tracers (e.g., Lequeux 2005; Gratier et al. 2013, see also the Leiden Atomic and Molecular Database ${ }^{2}$ ). Thus, $\mathrm{CN}, \mathrm{CS}, \mathrm{NC}_{3} \mathrm{~N}$, and $\mathrm{HNCO}$ emission only comes from the densest part of the cloud, while $\mathrm{CO}$ is emitted throughout the area. Dust continuum emission, as shown by the SPIRE images, also traces the densest part of the cloud.

\section{Discussion}

Based on its SED, HBC 722 used to be a normal T Tauri-type star in quiescence, except for a flux excess in the $2.2-8 \mu \mathrm{m}$ wavelength range compared to the Taurus median (Kóspál et al. 2011). This excess indicates a stronger-than-usual thermal emission from the inner disk, pointing to either a large illuminated surface area (strong disk flaring, inner envelope), or internal disk heating due to accretion. The fact that the excess is present already at wavelengths as short as the $K$-band suggests that the dust disk probably extended inward to the sublimation radius (0.06-0.07 au for a K7-M0 star). The optical and near-IR SED of the quiescent system was consistent with a reddened K7-type

\footnotetext{
2 http://home.strw.leidenuniv.nl/ moldata/
}

stellar photosphere shortward of the $K$-band (Miller et al. 2011, see also Sect.3.1), which shows that the accreting gas within the dust sublimation radius, if present, did not contribute significantly to the optical emission and formed an optically thin inner gas disk in quiescence.

We compared the quiescent SED (constructed in Sect. 3.1, representative of the source in 2006 March-October) with photometric observations obtained in 2010 May, just preceeding the rapid brightening of the source (Semkov et al. 2010; Miller et al. 2011, WISE, this work). Our analysis revealed that HBC 722 in this kickoff state was already significantly brighter than in quiescence. The light curves published by Semkov et al. (2010) and Miller et al. (2011) show that this gradual flux rise started about six months before the outburst. The brightening had similar amplitudes from $B$ to $R$, a possible minimum in the $I$ band, and larger amplitudes again from the $J$ band to $4.5 \mu \mathrm{m}$, almost 1.3 times higher than in the $V$ band.

Based on these colors, the optical brightening in the kickoff state is consistent with the appearance of a new component with a temperature similar to the stellar temperature. When fit with the accretion disk model of Eq. (1) (Fig. 2, bottom left panel), the temperature at the inner edge of the disk was $3830 \mathrm{~K}$, powered by an accretion rate of $M \dot{M}=2.5 \times 10^{-7} M_{\odot}^{2} \mathrm{yr}^{-1}$ (Fig. 3). The outer radius of the fitted accretion disk is $\sim 0.07 \mathrm{au}$, thus it resided within the sublimation radius of the dust disk. The mid-IR data points are higher than the fitted accretion disk model, suggesting increased thermal dust emission, possibly due to dust structures situated above the accretion disk surface (e.g., a vertical inner disk wall) at the dust sublimation radius, in accordance with its temperature. At later epochs, this excess is not visible and the accretion disk model reproduces the observed SEDs, indicating that these dust structures disappeared. Thus, it is tempting to speculate that in the kickoff state we might witness the material that is being accumulated before the outburst close to the sublimation radius, and which later fell onto the star during the 

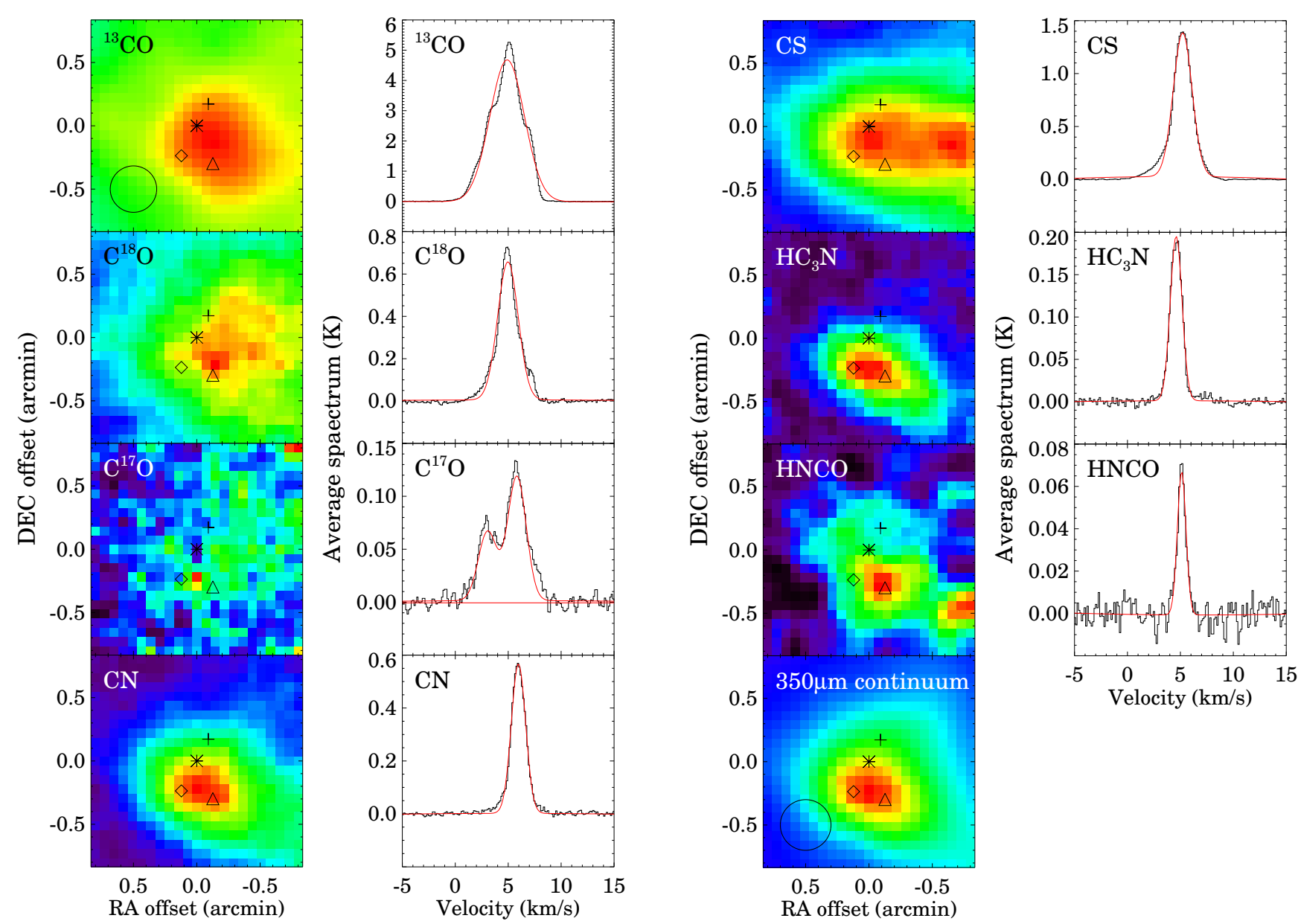

Fig. 7. Left: single-dish IRAM $30 \mathrm{~m}$ images of the area around HBC 722 in different lines, and a Herschel/SPIRE $350 \mu \mathrm{m}$ continuum image of the same area. For the IRAM data, the colors show the integrated line intensity, and the scale extends from 0 to $I_{\max }$, where $I_{\max }$ is $25.0,2.9,1.4$, $3.1,6.2,1.4$, and $0.25 \mathrm{~K} \mathrm{~km} \mathrm{~s}^{-1}$ for the ${ }^{13} \mathrm{CO} J=1-0, \mathrm{C}^{18} \mathrm{O} J=1-0, \mathrm{C}^{17} \mathrm{O} J=1-0, \mathrm{CN} N=1-0, \mathrm{CS} J=2-1, \mathrm{HC}_{3} \mathrm{~N} \mathrm{~J}=12-11$, and $\mathrm{HNCO}$ $J_{\mathrm{K}_{-1} \mathrm{~K}_{+1}}=5_{05}-4_{04}$ lines, respectively. For the Herschel image, the scale extends from 0 to $1050 \mathrm{MJy} \mathrm{sr}^{-1}$. The asterisk, plus, diamond, and triangle mark the positions of HBC 722, MMS1, MMS3, and MMS4, respectively. Circles in the bottom left corners of the top and bottom panels mark the beam sizes of the IRAM $30 \mathrm{~m}$ telescope and Herschel. Right: profiles of the lines averaged over the areas displayed in the left column (black histograms), with fitted Gaussian functions (red curves).

eruption. An event similar to the several months long preparatory phase of the HBC 722 outburst may be seen in the light curve of the latest FUor V960 Mon (Fig. 1 in Hackstein et al. 2015). If pre-outburst flux rise turns out to be a general feature of FUor outbursts, light curves obtained by all-sky surveys could be used to discover imminent FUor outbursts.

As described before in Sect. 3.1, the first brightness peak in the light curves was due to a short maximum in the accretion rate. Apparently, a packet of material arrived onto the star. The mass of the infalling material, estimated by integrating the accretion rate curve in Fig. 3, was $\sim 1.8 \times 10^{-6} M_{\odot}$. Interestingly, the accretion disk that reproduced the SED in the second column in Fig. 2 has an outer radius on the order of the dust sublimation radius. If we follow our speculation on the material that accumulated in the inner dust disk before the outburst, then the first peak in the light curves may be the consequence of the infall of the accumulated material onto the star. The freefall timescale at the dust sublimation radius is several days. Therefore it is plausible that this mass reservoir at the sublimation radius was emptied in a few weeks. The appearance of a short brightness maximum at the very beginning of the eruption is a characteristic feature of an outburst model family proposed by Bell et al. (1995). They postulate that eruptions are due to a thermal instability in the disk, and when the instability is triggered farther from the star than the inner edge of the disk, an ionization front, separating the cooler metastable and the hot high state areas, will propagate rapidly toward the star. Such an initial peak was observed in V1057 Cyg, for instance. In the case of HBC 722 the trigger could have occurred around the inner edge of the dust disk, where matter was piled up before the outburst.

During the following five months, between 2011 April and August, the brightness of HBC 722 stayed more or less constant. The fact that this flat part in the light curve started first in the infrared, where the first peak was weaker, and appeared only later in the $B$ band where the first peak was the strongest, suggests that here we simply see the overlap between two parts of the light curve: the decaying first peak and the emerging second brightness increase, which resulted in a transition period of approximately constant brightness. The light curve suggests that the process causing the first peak completely disappeared by mid-2011, and it is unclear from our data whether this process plays an essential role in the outburst mechanism.

The brightness rise between 2011 August and 2013 April is the most interesting part of the outburst. According to Fig. 3, the 
disk area participating in the outburst is expanding as its outer radius grows approximately linearly in time, from $\sim 0.07$ au to $\sim 0.15 \mathrm{au}$. This outward propagation resembles the predictions of the thermal instability model of Bell \& Lin (1994), where a hot, partially or fully ionized inner disk expands into the colder outer material, triggering a thermal instability at the ionization front that separates the two phases. Since this second ionization front propagates outward, its pace is significantly lower than the one during the first peak (see above). The linear increase of the radius of the accretion disk supports this hypothesis. The expansion of an ionization front stops at an equilibrium radius, where the ionization and recombination rates are equal, just like our data indicate. The expansion velocity of the outer radius of the accretion disk in Fig. 3 measured between 2011 August and 2013 April is on the order of $240 \mathrm{~m} \mathrm{~s}^{-1}$. This value is higher than the speed computed for a standard model of Bell \& Lin (1994) for an outward-propagating ionization front, determined by the time sufficient for mass transfer from the ionized region outward to increase the surface density enough for the thermal instability.

According to our modeling, the outward-expanding accretion disk extends over the sublimation radius of $0.07-0.08 \mathrm{au}$, thus the accretion disk may overlap with the dust disk. In the plateau phase, after 2013 April, the accretion rate reached a level even higher than the peak in 2010 September, and has been constant for several years. The total accreted mass in this phase is significantly higher than in the first peak. We predict that the plateau phase will end when the matter available in the inner disk is depleted. Assuming a simple disk model with a power-law surface density distribution of $\Sigma \sim r^{-1.5}$, and a total disk mass of $0.01 M_{\odot}$ (Sect. 3.3), the innermost region within 0.15 au contains enough mass to maintain the outburst for about 18 years. After the depletion of the inner disk the outburst stops, possibly leaving an extended, optically thin inner hole in the disk, which may be noticeable in the SED as a lack of mid-IR excess. Considering that we saw no indication of an inner hole in HBC 722 before the outburst, this hole probably fills up before the system can erupt again.

The accretion rate changes in the HBC 722 system during its outburst, as outlined by our measurements, can be compared with the conclusions drawn from multiepoch X-ray observations by Liebhart et al. (2014). The authors obtained three X-ray measurements, the first during the first peak of the light curve, the second during the local brightness minimum before the rebrightening, and the third during the plateau phase. They suggested that the first peak was due to an initial strong disk instability that rapidly led to enhanced accretion. It transported a huge amount of dust-free gas to the vicinity of the star, which absorbed all X-ray emission from the innermost region. This is consistent with our interpretation of the first brightness peak in the light curves in terms of a short maximum in the accretion rate. At their second X-ray epoch, Liebhart et al. (2014) could marginally detect the star, indicating significantly less gas around the star, in accordance with our picture where the first peak was followed by a rapid drop of the accretion rate. Finally, Liebhart et al. (2014) proposed that by their third epoch the accretion rate increased again, producing enhanced absorption. Our Fig. 3 shows that the accretion rate indeed increased by a factor of four between the dates of the second and third X-ray measurements.

If the present accretion rate remains, HBC 722 would accrete about $1 \times 10^{-4} M_{\odot}$ during the hypothesized length of the present outburst, 18 years. The disk mass, however, is relatively low, below $0.01 M_{\odot}$, which allows not more than 100 such eruptions to consume the whole circumstellar disk. A possible source of replenishment of material could be the flattened envelope of $0.03 M_{\odot}$, detected in molecular gas emission in Sect. 3.3. A detailed investigation of this structure, including the exploration of its infall pattern and mass infall rate, is indispensable to answer this question.

\section{Summary and conclusions}

Based on our optical-infrared monitoring, spectroscopic observations, and millimeter mapping, we performed a detailed study of the first six years of the outburst of the low-mass T Tauri star HBC 722. We interpreted the first brightness peak, lasting several months, as the rapid fall of piled-up material from the inner disk onto the star. This was followed by a monotonic flux rise, which can be explained by increasing accretion rate and emitting area. Our observations are consistent with the predictions of Bell et al. (1995) for a system where thermal instability is triggered at an intermediate radius within the metastable disk area. Their model predicts a rapid ionization from propagating toward the star, causing a short-lived brightness peak, similar to what we observed in 2010 September. In parallel, a second, slower ionization front starts to expand outward, which can be the physical reason for the re-brightening of HBC 722 after 2011 September. Our study of HBC 722 demonstrated that accretion-related outbursts can occur in young stellar objects even with very low-mass disks. Our results strengthen the theory that eruptive phenomena may appear throughout star formation from the embedded phase to the Class II phase, and that possibly all young stars undergo phases of temporarily increased accretion.

Acknowledgements. We thank the referee for the comments that greatly improved the paper and Z. Zhu for useful discussions on the accretion disk fitting. This work was supported by the Momentum grant of the MTA CSFK Lendület Disk Research Group, the Lendület grant LP2012-31 of the Hungarian Academy of Sciences, and the Hungarian Research Fund OTKA grant K101393. A.M. acknowledges support from the Bolyai Research Fellowship of the Hungarian Academy of Sciences. G.H. acknowledges support by the Ministry for the Economy, Development, and Tourism's Programa Iniciativa Milenio through grant IC 120009; by Proyecto Basal PFB-06/2007; and by CONICYT-PCHA/Doctorado Nacional grant 2014-63140099. The William Herschel Telescope and its service program are operated on the island of La Palma by the Isaac Newton Group in the Spanish Observatorio del Roque de los Muchachos of the Instituto de Astrofísica de Canarias. This work is based in part on observations made with the Telescopio Carlos Sanchez operated on the island of Tenerife by the Instituto de Astrofísica de Canarias in the Observatorio del Teide. The authors wish to thank the telescope manager A. Oscoz, the support astronomers and telescope operators for their help during the observations, as well as the service mode observers. Based on observations made with the Gran Telescopio Canarias (GTC), installed in the Spanish Observatorio del Roque de los Muchachos of the Instituto de Astrofísica de Canarias, in the island of La Palma. This work is partly based on observations carried out under project number VA6C with the IRAM Plateau de Bure Interferometer and under project number 260-11 with the IRAM $30 \mathrm{~m}$ Telescope. IRAM is supported by INSU/CNRS (France), MPG (Germany) and IGN (Spain). This work has benefited from research funding from the European Community's sixth Framework Programme under RadioNet R113CT 20035058187.

\section{References}

Acosta-Pulido, J. A., Kun, M., Ábrahám, P., et al. 2007, AJ, 133, 2020

Antoniucci, S., Arkharov, A., Klimanov, S., et al. 2013, ATel, 5023

Armond, T., Reipurth, B., Bally, J., \& Aspin, C. 2011, A\&A, 528, A125 Aspin, C., \& Reipurth, B. 2009, AJ, 138, 1137

Audard, M., Ábrahám, P., Dunham, M. M., et al. 2014, in Protostars and Planets VI, eds. H. Beuther, R. S. Klessen, C. P. Dullemond, \& T. Henning (Tucson: University of Arizona Press), 387

Baek, G., Pak, S., Green, J. D., et al. 2015, AJ, 149, 73

Bell, K. R., \& Lin, D. N. C. 1994, ApJ, 427, 987

Bell, K. R., Lin, D. N. C., Hartmann, L. W., \& Kenyon, S. J. 1995, ApJ, 444, 376 
Castelli, F., \& Kurucz, R. L. 2004, AU Symp., 210, poster A20

Cepa, J. 2010, in Highlights of Spanish Astrophysics V, eds. J. M. Diego, L. J. Goicoechea, J. I. González-Serrano, \& J. Gorgas, 15

Cepa, J., Aguiar-Gonzalez, M., Bland-Hawthorn, J., et al. 2003, in Instrument Design and Performance for Optical/Infrared Ground-based Telescopes, eds. M. Iye, \& A. F. M. Moorwood, SPIE Conf. Ser., 4841, 1739

Cohen, M., \& Kuhi, L. V. 1979, ApJS, 41, 743

Dunham, M. M., Arce, H. G., Bourke, T. L., et al. 2012, ApJ, 755, 157

Gramajo, L. V., Rodón, J. A., \& Gómez, M. 2014, AJ, 147, 140

Gratier, P., Pety, J., Guzmán, V., et al. 2013, A\&A, 557, A101

Green, J. D., Evans, II, N. J., Kóspál, Á., et al. 2011, ApJ, 731, L25

Green, J. D., Evans, II, N. J., Kóspál, Á., et al. 2013a, ApJ, 772, 117

Green, J. D., Robertson, P., Baek, G., et al. 2013b, ApJ, 764, 22

Hackstein, M., Haas, M., Kóspál, Á., et al. 2015, A\&A, 582, L12

Hartmann, L., \& Kenyon, S. J. 1996, ARA\&A, 34, 207

Herbig, G. H. 1977, ApJ, 217, 693

Kenyon, S. J., Hartmann, L. W., Strom, K. M., \& Strom, S. E. 1990, AJ, 99, 869

Kóspál, Á., Ábrahám, P., Acosta-Pulido, J. A., et al. 2011, A\&A, 527, A133
Kun, M., Szegedi-Elek, E., Moór, A., et al. 2011, MNRAS, 413, 2689

Lee, J.-E., Park, S., Green, J. D., et al. 2015, ApJ, 807, 84

Lequeux, J. 2005, The Interstellar Medium (Springer)

Liebhart, A., Güdel, M., Skinner, S. L., \& Green, J. 2014, A\&A, 570, L11

Lorenzetti, D., Efimova, N., Larionov, V., et al. 2012, ATel, 4123, 1

Miller, A. A., Hillenbrand, L. A., Covey, K. R., et al. 2011, ApJ, 730, 80

Rebull, L. M., Guieu, S., Stauffer, J. R., et al. 2011, ApJS, 193, 25

Ricci, L., Testi, L., Natta, A., \& Brooks, K. J. 2010, A\&A, 521, A66

Semkov, E., \& Peneva, S. 2010, ATel, 2801, 1

Semkov, E. H., Peneva, S. P., Munari, U., Milani, A., \& Valisa, P. 2010, A\&A, 523, L3

Semkov, E. H., Peneva, S. P., Munari, U., et al. 2012, A\&A, 542, A43

Semkov, E. H., Peneva, S. P., Ibryamov, S. I., \& Dimitrov, D. P. 2014, Bulgarian Astron. J., 20, 59

Siess, L., Dufour, E., \& Forestini, M. 2000, A\&A, 358, 593

Sung, H.-I., Park, W.-K., Yang, Y., et al. 2013, J. Korean Astron. Soc., 46, 253

Wilson, T. L., \& Rood, R. 1994, ARA\&A, 32, 191

Wright, E. L., Eisenhardt, P. R. M., Mainzer, A. K., et al. 2010, AJ, 140, 1868

Zhu, Z., Hartmann, L., Calvet, N., et al. 2007, ApJ, 669, 483 


\section{Appendix A: Additional tables}

Table A.1. Optical and near-IR photometry in magnitudes for HBC 722.

\begin{tabular}{|c|c|c|c|c|c|c|c|c|c|}
\hline Date & $\mathrm{JD}-2400000$ & $B$ & $V$ & $R$ & $I$ & $J$ & $H$ & $K_{\mathrm{S}}$ & Telescope \\
\hline $2010-12-03$ & 55534.34 & & & & & $10.36(1)$ & $9.41(1)$ & $8.90(1)$ & TCS \\
\hline 2011-03-24 & 55644.63 & $16.58(3)$ & $14.95(2)$ & $13.87(2)$ & $12.68(1)$ & & & & Schmidt \\
\hline $2011-03-25$ & 55645.56 & & $14.92(2)$ & $13.83(1)$ & $12.63(1)$ & & & & RCC \\
\hline $2011-03-30$ & 55650.59 & & $15.01(1)$ & $13.85(1)$ & $12.66(1)$ & & & & $\mathrm{RCC}$ \\
\hline 2011-03-31 & 55651.58 & $16.74(6)$ & $14.99(2)$ & $13.87(1)$ & $12.65(1)$ & & & & RCC \\
\hline 2011-04-10 & 55661.55 & $16.48(5)$ & $14.93(5)$ & $13.82(1)$ & $12.66(2)$ & & & & Schmidt \\
\hline 2011-04-23 & 55674.55 & & $15.09(1)$ & 13.91(1) & $12.70(1)$ & & & & RCC \\
\hline $2011-04-27$ & 55678.65 & & $15.00(1)$ & $13.85(1)$ & & & & & IAC80 \\
\hline 2011-04-27 & 55678.70 & & & & & $10.72(1)$ & $9.70(1)$ & $9.11(1)$ & TCS \\
\hline $2011-05-26$ & 55707.52 & & $15.03(1)$ & $13.87(1)$ & $12.66(1)$ & & & & $\mathrm{RCC}$ \\
\hline 2011-06-05 & 55717.52 & & $15.17(1)$ & $13.98(1)$ & $12.65(2)$ & & & & RCC \\
\hline 2011-06-19 & 55732.38 & $16.86(19)$ & $14.99(2)$ & $13.85(1)$ & $12.62(4)$ & & & & Schmidt \\
\hline $2011-06-20$ & 55733.42 & $16.91(22)$ & $14.97(8)$ & $13.78(1)$ & $12.61(2)$ & & & & Schmidt \\
\hline $2011-06-26$ & 55739.48 & $16.55(4)$ & $14.85(2)$ & $13.68(1)$ & $12.47(1)$ & & & & $\mathrm{RCC}$ \\
\hline $2011-07-21$ & 55763.62 & & & & & $10.65(2)$ & $9.67(3)$ & $9.17(1)$ & WHT/LIRIS \\
\hline 2011-08-04 & 55777.61 & & & & & $10.60(1)$ & $9.60(1)$ & $9.03(1)$ & TCS \\
\hline 2011-08-05 & 55779.34 & $16.71(4)$ & $14.95(1)$ & $13.81(1)$ & $12.60(2)$ & & & & Schmidt \\
\hline 2011-08-09 & 55782.63 & & & & & $10.57(1)$ & $9.59(1)$ & $9.00(1)$ & TCS \\
\hline 2011-08-11 & 55785.38 & $16.75(5)$ & $15.03(2)$ & $13.87(3)$ & $12.64(1)$ & & & & Schmidt \\
\hline 2011-08-12 & 55786.36 & $16.35(17)$ & $14.94(7)$ & $13.80(1)$ & $12.60(2)$ & & & & Schmidt \\
\hline $2011-08-14$ & 55788.33 & $16.86(27)$ & 14.91(19) & $13.79(4)$ & $12.56(3)$ & & & & Schmidt \\
\hline $2011-08-15$ & 55789.31 & $16.66(12)$ & $14.98(3)$ & $13.75(2)$ & $12.57(8)$ & & & & Schmidt \\
\hline $2011-08-15$ & 55789.43 & & & & & $10.55(3)$ & $9.60(1)$ & $9.00(1)$ & TCS \\
\hline 2011-08-17 & 55791.41 & $16.69(8)$ & $14.91(1)$ & $13.73(1)$ & $12.54(1)$ & & & & Schmidt \\
\hline 2011-08-19 & 55792.56 & $16.73(5)$ & $14.89(4)$ & $13.74(5)$ & $12.59(1)$ & & & & Schmidt \\
\hline $2011-08-20$ & 55794.49 & $16.65(3)$ & $14.91(2)$ & $13.74(2)$ & $12.57(2)$ & & & & Schmidt \\
\hline $2011-08-24$ & 55797.57 & $16.59(17)$ & $14.89(3)$ & $13.71(1)$ & $12.53(1)$ & & & & Schmidt \\
\hline $2011-08-25$ & 55799.36 & $16.74(04)$ & $14.98(1)$ & $13.79(1)$ & $12.57(1)$ & & & & RCC \\
\hline 2011-08-29 & 55803.37 & 16.67(9) & $14.98(1)$ & $13.81(1)$ & $12.59(1)$ & & & & $\mathrm{RCC}$ \\
\hline $2011-08-30$ & 55804.39 & $16.71(2)$ & $14.95(1)$ & $13.79(1)$ & $12.58(1)$ & & & & RCC \\
\hline 2011-09-03 & 55807.51 & $16.60(5)$ & $15.02(1)$ & $13.85(1)$ & $12.67(1)$ & & & & Schmidt \\
\hline 2011-09-06 & 55811.30 & $16.78(6)$ & $15.04(3)$ & $13.90(1)$ & $12.65(1)$ & & & & Schmidt \\
\hline 2011-09-09 & 55813.63 & & & & & $10.62(1)$ & $9.60(1)$ & $9.01(1)$ & TCS \\
\hline 2011-09-09 & 55814.36 & 17.07(20) & $15.07(7)$ & $13.98(12)$ & $12.73(4)$ & & & & Schmidt \\
\hline 2011-09-09 & 55814.43 & & & & & $10.60(1)$ & $9.61(1)$ & $9.04(2)$ & TCS \\
\hline 2011-09-11 & 55816.43 & & & & & $10.59(1)$ & $9.59(1)$ & $9.04(2)$ & TCS \\
\hline 2011-09-12 & 55817.46 & & & & & $10.60(1)$ & $9.61(1)$ & $9.03(1)$ & TCS \\
\hline 2011-09-13 & 55818.53 & & & & & $10.61(1)$ & $9.56(1)$ & $9.00(1)$ & TCS \\
\hline $2011-09-16$ & 55821.33 & $16.66(4)$ & $15.00(2)$ & $13.78(2)$ & $12.51(1)$ & & & & Schmidt \\
\hline 2011-09-17 & 55822.32 & $16.75(5)$ & $14.94(4)$ & $13.77(2)$ & $12.54(3)$ & & & & Schmidt \\
\hline 2011-09-21 & 55826.34 & $16.56(2)$ & $14.92(3)$ & $13.77(1)$ & $12.57(1)$ & & & & Schmidt \\
\hline 2011-09-24 & 55829.32 & $16.75(3)$ & $14.96(1)$ & $13.78(1)$ & $12.57(1)$ & & & & RCC \\
\hline $2011-09-25$ & 55830.28 & $16.53(12)$ & $14.80(3)$ & $13.75(4)$ & $12.47(2)$ & & & & Schmidt \\
\hline $2011-09-25$ & 55830.33 & $16.74(2)$ & $14.95(1)$ & $13.77(1)$ & $12.53(1)$ & & & & $\mathrm{RCC}$ \\
\hline $2011-09-26$ & 55831.33 & $16.80(2)$ & $15.01(1)$ & 13.81(1) & $12.53(1)$ & & & & $\mathrm{RCC}$ \\
\hline $2011-10-07$ & 55842.41 & & & & & $10.50(1)$ & $9.51(1)$ & $8.94(1)$ & TCS \\
\hline 2011-10-08 & 55843.41 & & & & & $10.50(1)$ & $9.51(1)$ & $8.92(2)$ & TCS \\
\hline 2011-10-11 & 55846.44 & & & & & $10.46(1)$ & $9.52(1)$ & $9.00(2)$ & TCS \\
\hline $2011-10-26$ & 55861.24 & $16.46(19)$ & $14.80(8)$ & $13.60(3)$ & $12.43(2)$ & & & & Schmidt \\
\hline $2011-10-27$ & 55862.28 & $16.54(8)$ & $14.62(16)$ & $13.68(2)$ & $12.38(6)$ & & & & Schmidt \\
\hline $2011-10-27$ & 55862.46 & & & & & $10.42(1)$ & $9.44(2)$ & $8.84(3)$ & TCS \\
\hline 2011-10-28 & 55863.20 & $16.34(5)$ & $14.69(2)$ & $13.56(1)$ & $12.41(1)$ & & & & Schmidt \\
\hline $2011-10-29$ & 55864.42 & $16.32(7)$ & $14.71(5)$ & $13.51(2)$ & $12.35(1)$ & & & & Schmidt \\
\hline $2011-10-30$ & 55865.40 & $16.36(2)$ & $14.64(1)$ & $13.55(1)$ & $12.40(1)$ & & & & Schmidt \\
\hline $2011-10-31$ & 55866.40 & $16.39(3)$ & $14.62(2)$ & $13.53(1)$ & $12.37(2)$ & & & & Schmidt \\
\hline 2011-10-31 & 55866.40 & & & & & $10.39(1)$ & $9.43(1)$ & $8.89(1)$ & TCS \\
\hline
\end{tabular}


Table A.1. continued.

\begin{tabular}{|c|c|c|c|c|c|c|c|c|c|}
\hline Date & $\mathrm{JD}-2400000$ & B & $V$ & $R$ & $I$ & $J$ & $H$ & $K_{\mathrm{S}}$ & Telescope \\
\hline 2011-11-01 & 55867.23 & $16.40(8)$ & $14.71(2)$ & $13.54(1)$ & $12.40(2)$ & & & & Schmidt \\
\hline 2011-11-01 & 55867.39 & & & & & $10.41(1)$ & $9.41(1)$ & $8.89(1)$ & TCS \\
\hline 2011-11-02 & 55868.22 & $16.41(2)$ & $14.68(1)$ & $13.52(1)$ & $12.38(2)$ & & & & Schmidt \\
\hline 2011-11-02 & 55868.37 & & & & & $10.42(1)$ & $9.45(1)$ & $8.90(1)$ & TCS \\
\hline 2011-11-03 & 55869.39 & $16.30(11)$ & $14.61(22)$ & $13.66(10)$ & $12.50(8)$ & & & & Schmidt \\
\hline 2011-11-04 & 55870.39 & & & & & $10.40(1)$ & $9.44(1)$ & $8.90(1)$ & TCS \\
\hline 2011-11-05 & 55871.37 & & & & & $10.41(1)$ & $9.46(1)$ & $8.89(1)$ & TCS \\
\hline 2011-11-06 & 55872.42 & & & & & $10.38(1)$ & $9.44(1)$ & $8.90(1)$ & $\mathrm{TCS}$ \\
\hline 2011-11-08 & 55874.38 & & & & & $10.35(1)$ & $9.42(1)$ & $8.90(1)$ & TCS \\
\hline 2011-11-13 & 55879.26 & & $14.69(1)$ & $13.51(3)$ & $12.35(1)$ & & & & RCC \\
\hline 2011-11-22 & 55888.19 & $16.22(19)$ & $14.60(3)$ & $13.44(3)$ & $12.32(3)$ & & & & Schmidt \\
\hline $2011-11-23$ & 55889.18 & $16.23(5)$ & $14.60(1)$ & $13.51(2)$ & $12.32(1)$ & & & & Schmidt \\
\hline $2011-11-24$ & 55890.18 & $16.25(2)$ & $14.56(3)$ & $13.45(1)$ & $12.28(1)$ & & & & Schmidt \\
\hline $2011-11-25$ & 55891.22 & $16.22(8)$ & $14.58(3)$ & $13.46(3)$ & $12.32(2)$ & & & & Schmidt \\
\hline $2011-11-27$ & 55893.28 & $16.23(3)$ & $14.61(1)$ & $13.46(2)$ & $12.32(1)$ & & & & Schmidt \\
\hline $2011-11-28$ & 55894.18 & $16.29(1)$ & $14.64(4)$ & $13.45(1)$ & 12.31(1) & & & & Schmidt \\
\hline $2011-11-29$ & 55895.18 & $16.34(8)$ & $14.72(1)$ & $13.49(1)$ & $12.32(2)$ & & & & Schmidt \\
\hline 2011-11-29 & 55895.32 & & & & & $10.31(5)$ & $9.35(1)$ & $8.83(1)$ & TCS \\
\hline 2011-11-30 & 55896.18 & $16.20(9)$ & $14.57(1)$ & $13.41(2)$ & $12.22(1)$ & & & & Schmidt \\
\hline 2011-11-30 & 55896.32 & & & & & $10.30(1)$ & $9.34(1)$ & $8.84(1)$ & TCS \\
\hline 2011-12-31 & 55927.18 & $16.09(7)$ & $14.31(7)$ & $13.24(1)$ & $12.09(1)$ & & & & Schmidt \\
\hline 2012-01-05 & 55932.32 & $16.01(2)$ & $14.29(1)$ & $13.13(1)$ & 12.01(1) & & & & IAC80 \\
\hline 2012-01-12 & 55939.31 & & & & & $10.10(1)$ & $9.16(1)$ & $8.63(1)$ & TCS \\
\hline 2012-01-12 & 55939.31 & & & & $11.95(1)$ & & & & IAC80 \\
\hline 2012-02-10 & 55968.22 & $16.12(14)$ & $14.26(5)$ & $13.01(3)$ & $11.89(2)$ & & & & Schmidt \\
\hline $2012-04-28$ & 56045.59 & $15.88(7)$ & $14.04(6)$ & $12.97(2)$ & 11.84(1) & & & & Schmidt \\
\hline $2012-05-20$ & 56067.51 & $15.60(4)$ & $14.01(1)$ & $12.90(1)$ & 11.81(1) & & & & RCC \\
\hline $2012-05-24$ & 56071.51 & $16.04(13)$ & $14.02(1)$ & $12.91(1)$ & $11.83(1)$ & & & & RCC \\
\hline 2012-06-01 & 56079.52 & $15.57(8)$ & $13.92(5)$ & $12.97(2)$ & $11.87(3)$ & & & & Schmidt \\
\hline 2012-06-07 & 56086.46 & $15.72(10)$ & $14.01(5)$ & $12.95(3)$ & $11.81(2)$ & & & & Schmidt \\
\hline 2012-06-19 & 56098.49 & $15.64(7)$ & $13.95(8)$ & $12.77(2)$ & $11.72(1)$ & & & & Schmidt \\
\hline 2012-07-22 & 56131.32 & $15.86(24)$ & $13.89(10)$ & $12.85(2)$ & $11.70(1)$ & & & & Schmidt \\
\hline $2012-07-23$ & 56132.32 & $15.23(25)$ & $13.94(7)$ & $12.79(1)$ & $11.60(8)$ & & & & Schmidt \\
\hline 2012-07-25 & 56134.41 & $15.78(5)$ & $13.85(2)$ & $12.72(1)$ & $11.57(1)$ & & & & IAC80 \\
\hline $2012-07-25$ & 56134.58 & & & & & $9.82(1)$ & $8.92(1)$ & $8.38(2)$ & TCS \\
\hline 2012-08-09 & 56149.37 & $15.43(1)$ & $13.83(1)$ & $12.72(1)$ & $11.63(1)$ & & & & $\mathrm{RCC}$ \\
\hline 2012-08-19 & 56159.40 & $15.33(2)$ & $13.74(1)$ & $12.64(1)$ & $11.57(1)$ & & & & $\mathrm{RCC}$ \\
\hline $2012-08-20$ & 56160.41 & $16.31(5)$ & $13.99(3)$ & $12.80(4)$ & $11.62(1)$ & & & & IAC80 \\
\hline 2012-08-20 & 56160.70 & & & & & $9.80(2)$ & $8.84(1)$ & $8.36(1)$ & TCS \\
\hline $2012-08-25$ & 56164.37 & $15.43(4)$ & $13.79(3)$ & $12.71(2)$ & $11.61(2)$ & & & & $\mathrm{RCC}$ \\
\hline $2012-08-26$ & 56165.33 & $15.14(32)$ & $13.84(2)$ & $12.71(2)$ & $11.67(2)$ & & & & $\mathrm{RCC}$ \\
\hline $2012-09-16$ & 56187.36 & & $13.73(1)$ & $12.66(1)$ & $11.61(2)$ & & & & $\mathrm{RCC}$ \\
\hline 2012-10-09 & 56210.39 & & & & & $9.82(2)$ & $8.85(1)$ & $8.26(1)$ & TCS \\
\hline $2012-10-10$ & 56211.44 & & & & & $9.78(1)$ & 8.81(1) & $8.25(1)$ & TCS \\
\hline 2012-10-11 & 56212.43 & & & & & $9.73(1)$ & $8.79(1)$ & $8.21(1)$ & TCS \\
\hline $2012-10-13$ & 56214.43 & & & & & $9.71(1)$ & $8.75(1)$ & $8.23(1)$ & TCS \\
\hline 2013-07-24 & 56497.54 & & $13.56(5)$ & $12.27(2)$ & $11.24(2)$ & & & & Schmidt \\
\hline 2013-08-08 & 56513.36 & $14.83(3)$ & $13.30(1)$ & $12.26(1)$ & $11.18(2)$ & & & & Schmidt \\
\hline $2013-08-17$ & 56522.41 & $14.94(7)$ & $13.38(3)$ & $12.30(1)$ & $11.25(1)$ & & & & Schmidt \\
\hline 2013-08-31 & 56536.34 & $15.24(20)$ & $13.39(2)$ & $12.31(1)$ & 11.21(1) & & & & $\mathrm{RCC}$ \\
\hline 2013-09-01 & 56537.41 & $15.05(3)$ & $13.42(8)$ & $12.32(1)$ & $11.25(1)$ & & & & $\mathrm{RCC}$ \\
\hline 2013-09-03 & 56539.45 & $15.08(20)$ & $13.37(3)$ & $12.34(1)$ & $11.25(1)$ & & & & $\mathrm{RCC}$ \\
\hline 2013-09-04 & 56540.33 & $15.30(20)$ & 13.61(18) & $12.33(4)$ & $11.26(2)$ & & & & RCC \\
\hline $2014-02-24$ & 56712.66 & $15.03(4)$ & $13.38(4)$ & $12.33(3)$ & $11.29(3)$ & & & & Schmidt \\
\hline 2014-04-08 & 56755.61 & $14.99(6)$ & $13.33(5)$ & $12.27(2)$ & $11.24(3)$ & & & & Schmidt \\
\hline 2014-04-30 & 56777.58 & $15.08(6)$ & $13.45(3)$ & $12.32(4)$ & $11.24(4)$ & & & & Schmidt \\
\hline 2014-06-05 & 56813.53 & $15.02(7)$ & $13.53(3)$ & $12.41(3)$ & $11.34(3)$ & & & & Schmidt \\
\hline 2015-01-06 & 57029.22 & $14.98(6)$ & $13.42(5)$ & $12.26(3)$ & $11.18(3)$ & & & & Schmidt \\
\hline
\end{tabular}


Table A.1. continued.

\begin{tabular}{|c|c|c|c|c|c|c|c|c|c|}
\hline Date & $\mathrm{JD}-2400000$ & $B$ & $V$ & $R$ & $I$ & $J$ & $H$ & $K_{\mathrm{S}}$ & Telescope \\
\hline $2015-02-17$ & 57070.68 & $15.21(14)$ & $13.36(5)$ & $12.34(2)$ & $11.16(2)$ & & & & Schmidt \\
\hline 2015-02-18 & 57071.68 & $15.12(6)$ & $13.37(5)$ & $12.27(2)$ & $11.20(2)$ & & & & Schmidt \\
\hline $2015-03-17$ & 57098.64 & $15.00(4)$ & $13.30(5)$ & $12.25(1)$ & $11.13(2)$ & & & & Schmidt \\
\hline 2015-04-09 & 57121.62 & $14.88(19)$ & $13.57(7)$ & $12.28(1)$ & $11.19(3)$ & & & & Schmidt \\
\hline 2015-04-13 & 57125.59 & $14.99(6)$ & $13.35(2)$ & $12.33(4)$ & $11.26(3)$ & & & & Schmidt \\
\hline 2015-04-20 & 57132.53 & $14.87(16)$ & $13.38(3)$ & $12.30(2)$ & $11.22(2)$ & & & & Schmidt \\
\hline $2015-04-23$ & 57135.57 & $15.07(5)$ & $13.39(5)$ & $12.26(1)$ & $11.20(2)$ & & & & Schmidt \\
\hline 2015-05-08 & 57150.51 & $15.16(8)$ & $13.58(3)$ & $12.41(3)$ & $11.28(4)$ & & & & Schmidt \\
\hline $2015-05-16$ & 57159.44 & $15.12(14)$ & $13.33(9)$ & $12.31(2)$ & $11.25(4)$ & & & & Schmidt \\
\hline $2015-05-20$ & 57163.72 & & & & & $9.30(1)$ & $8.35(1)$ & $7.79(1)$ & TCS \\
\hline $2015-05-21$ & 57164.78 & & & & & $9.30(1)$ & $8.39(3)$ & $7.81(16)$ & TCS \\
\hline $2015-06-01$ & 57174.54 & $15.03(10)$ & $13.34(2)$ & $12.27(1)$ & $11.20(1)$ & & & & Schmidt \\
\hline 2015-06-01 & 57175.46 & $15.01(10)$ & $13.38(4)$ & $12.26(3)$ & $11.18(2)$ & & & & Schmidt \\
\hline $2015-06-02$ & 57176.49 & $15.06(3)$ & $13.33(4)$ & $12.28(1)$ & $11.21(2)$ & & & & Schmidt \\
\hline 2015-06-03 & 57177.44 & 15.08(10) & $13.51(7)$ & $12.30(1)$ & $11.20(1)$ & & & & Schmidt \\
\hline $2015-06-06$ & 57180.49 & $14.96(8)$ & $13.41(3)$ & $12.32(3)$ & $11.21(4)$ & & & & Schmidt \\
\hline $2015-06-07$ & 57181.48 & $15.15(10)$ & $13.46(6)$ & $12.31(4)$ & $11.23(2)$ & & & & Schmidt \\
\hline 2015-06-12 & 57186.45 & $14.83(8)$ & $13.35(1)$ & $12.26(3)$ & $11.18(1)$ & & & & Schmidt \\
\hline $2015-06-18$ & 57191.52 & $15.10(10)$ & $13.33(2)$ & $12.27(1)$ & $11.16(1)$ & & & & Schmidt \\
\hline $2015-07-16$ & 57220.49 & $14.90(4)$ & $13.31(2)$ & $12.21(1)$ & $11.15(1)$ & & & & Schmidt \\
\hline $2015-07-22$ & 57226.43 & $14.89(3)$ & $13.42(2)$ & $12.35(1)$ & $11.26(3)$ & & & & Schmidt \\
\hline $2015-08-05$ & 57240.36 & $14.93(2)$ & $13.39(2)$ & $12.33(1)$ & $11.24(1)$ & & & & Schmidt \\
\hline $2015-08-12$ & 57247.32 & $14.98(13)$ & $13.30(6)$ & $12.28(4)$ & $11.16(3)$ & & & & Schmidt \\
\hline 2015-08-31 & 57266.37 & $14.91(22)$ & $13.42(11)$ & $12.34(7)$ & $11.23(5)$ & & & & Schmidt \\
\hline 2015-09-01 & 57267.33 & $15.12(35)$ & $13.40(1)$ & $12.30(3)$ & $11.24(1)$ & & & & Schmidt \\
\hline 2015-09-02 & 57268.32 & $15.03(49)$ & $13.35(4)$ & $12.26(1)$ & $11.21(2)$ & & & & Schmidt \\
\hline $2015-09-10$ & 57275.56 & $15.02(30)$ & $13.30(4)$ & $12.21(3)$ & $11.06(2)$ & & & & Schmidt \\
\hline $2015-09-15$ & 57281.33 & & $13.37(2)$ & $12.27(4)$ & $11.21(2)$ & & & & Schmidt \\
\hline 2015-09-17 & 57283.25 & & $13.36(4)$ & $12.31(3)$ & $11.22(1)$ & & & & Schmidt \\
\hline $2015-09-18$ & 57284.25 & & $13.42(10)$ & $12.39(5)$ & $11.21(3)$ & & & & Schmidt \\
\hline $2015-10-22$ & 57318.25 & & $13.52(4)$ & $12.45(2)$ & $11.32(2)$ & & & & Schmidt \\
\hline $2015-10-27$ & 57323.32 & & $13.39(4)$ & $12.34(2)$ & $11.23(3)$ & & & & Schmidt \\
\hline 2015-11-05 & 57332.19 & & $13.44(4)$ & $12.28(5)$ & $11.30(4)$ & & & & Schmidt \\
\hline 2015-11-08 & 57335.20 & & $13.46(2)$ & $12.42(3)$ & $11.29(1)$ & & & & Schmidt \\
\hline $2015-11-12$ & 57339.31 & & & $12.34(1)$ & $11.22(2)$ & & & & Schmidt \\
\hline 2016-03-10 & 57457.63 & $14.95(9)$ & $13.30(5)$ & $12.44(7)$ & $11.24(2)$ & & & & Schmidt \\
\hline $2016-04-03$ & 57481.62 & $15.24(12)$ & $13.32(5)$ & $12.30(3)$ & $11.19(6)$ & & & & Schmidt \\
\hline 2016-04-30 & 57508.56 & $15.05(4)$ & $13.41(3)$ & $12.32(1)$ & $11.20(1)$ & & & & Schmidt \\
\hline $2016-05-07$ & 57515.53 & $14.94(6)$ & $13.30(2)$ & $12.22(4)$ & $11.15(2)$ & & & & Schmidt \\
\hline $2016-05-21$ & 57530.44 & $15.40(7)$ & $13.52(2)$ & $12.32(2)$ & $11.29(2)$ & & & & Schmidt \\
\hline $2016-05-29$ & 57538.49 & $15.18(7)$ & $13.48(2)$ & $12.25(4)$ & $11.29(6)$ & & & & Schmidt \\
\hline 2016-05-31 & 57539.54 & $14.97(13)$ & $13.45(3)$ & $12.37(1)$ & $11.29(1)$ & & & & Schmidt \\
\hline 2016-06-08 & 57548.46 & $14.96(6)$ & $13.30(2)$ & $12.27(1)$ & 11.19(1) & & & & Schmidt \\
\hline $2016-06-23$ & 57563.46 & $14.96(9)$ & $13.37(9)$ & $12.26(3)$ & $11.20(2)$ & & & & Schmidt \\
\hline 2016-07-06 & 57576.45 & $15.05(4)$ & $13.43(3)$ & $12.33(4)$ & $11.25(1)$ & & & & Schmidt \\
\hline 2016-07-12 & 57581.54 & $15.15(5)$ & $13.45(1)$ & $12.34(2)$ & $11.27(3)$ & & & & Schmidt \\
\hline
\end{tabular}


Á. Kóspál et al.: Multiwavelength study of HBC 722

Table A.2. Spitzer/IRAC and WISE photometry for HBC 722. Fluxes are not color-corrected.

\begin{tabular}{|c|c|c|c|c|c|c|}
\hline Date & $\mathrm{JD}-2400000$ & $F_{3.4}(\mathrm{Jy})$ & $F_{3.6}(\mathrm{Jy})$ & $F_{4.5}(\mathrm{Jy})$ & $F_{4.6}(\mathrm{Jy})$ & Telescope \\
\hline $2010-05-29$ & 55346.30 & $0.082 \pm 0.004$ & & & $0.098 \pm 0.003$ & WISE \\
\hline $2010-11-26$ & 55527.10 & $0.168 \pm 0.005$ & & & $0.169 \pm 0.008$ & WISE \\
\hline 2011-09-08 & 55813.02 & & $0.127 \pm 0.004$ & $0.112 \pm 0.003$ & & Spitzer \\
\hline 2011-09-24 & 55829.49 & & $0.129 \pm 0.004$ & $0.115 \pm 0.004$ & & Spitzer \\
\hline $2011-11-29$ & 55895.31 & & $0.154 \pm 0.005$ & $0.136 \pm 0.004$ & & Spitzer \\
\hline 2012-01-06 & 55932.50 & & $0.169 \pm 0.005$ & $0.152 \pm 0.005$ & & Spitzer \\
\hline $2012-01-11$ & 55938.10 & & $0.173 \pm 0.005$ & $0.154 \pm 0.005$ & & Spitzer \\
\hline $2012-07-25$ & 56134.09 & & $0.240 \pm 0.007$ & $0.216 \pm 0.006$ & & Spitzer \\
\hline 2012-08-20 & 56160.46 & & $0.248 \pm 0.008$ & $0.226 \pm 0.007$ & & Spitzer \\
\hline $2012-09-16$ & 56186.75 & & $0.269 \pm 0.008$ & $0.247 \pm 0.008$ & & Spitzer \\
\hline $2012-10-12$ & 56212.64 & & $0.272 \pm 0.008$ & $0.248 \pm 0.007$ & & Spitzer \\
\hline
\end{tabular}

Table A.3. IRAM $2.7 \mathrm{~mm}$ photometry for HBC 722 and for the millimeter sources in its vicinity.

\begin{tabular}{ccccc}
\hline \hline Name & Peak flux $(\mathrm{mJy} /$ beam $)$ & Total flux $(\mathrm{mJy})$ & Size $\left(^{\prime \prime}\right)$ & PA $\left(^{\circ}\right)$ \\
\hline HBC 722 & $<0.08$ & $<0.08$ & $\ldots$ & $\ldots$ \\
MMS1 & $3.63 \pm 0.11$ & $4.53 \pm 0.22$ & $1.3 \times 1.1$ & 115 \\
MMS2 & $1.44 \pm 0.10$ & $1.61 \pm 0.18$ & $1.1 \times 0.6$ & 100 \\
MMS3 & $1.38 \pm 0.11$ & $4.03 \pm 0.40$ & $4.9 \times 2.0$ & 48 \\
MMS4 & $2.07 \pm 0.21$ & $4.34 \pm 0.61$ & $3.4 \times 1.6$ & 31 \\
MMS5 & $0.83 \pm 0.10$ & $2.58 \pm 0.30$ & $6.1 \times 1.8$ & 83 \\
MMS6 & $0.39 \pm 0.05$ & $0.68 \pm 0.13$ & $3.4 \times 0.2$ & 48 \\
MMS7 & $<0.08$ & $<0.08$ & $\ldots$ & $\ldots$ \\
\hline
\end{tabular}

